a cura del

Comitato Nazionale per il bicentenario della nascita di Antonio Meucci

Luigi Respighi

Per la priorità di

Antonio Meucci

nell'invenzione

del telefono

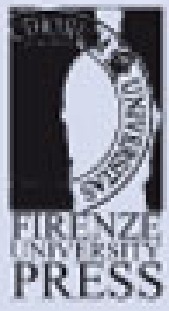


FONTI STORICHE E LETTERARIE

EDIZIONI CARTACEE E DIGITALI 



\title{
Luigi Respighi
}

\section{Per la priorità di Antonio Meucci nell'invenzione del telefono}

\author{
a cura del \\ Comitato Nazionale per il bicentenario \\ della nascita di Antonio Meucci
}

Firenze University Press

2008 
Per la priorità di Antonio Meucci nell'invenzione del telefono / Luigi Respighi ; a cura del Comitato Nazionale per il bicentenario della nascita di Antonio Meucci.

- Firenze : Firenze University Press, 2008.

(Fonti storiche e letterarie. Edizioni cartacee e digitali)

http://digital.casalini.it/9788884537591

ISBN 978-88-8453-759-1 (online)

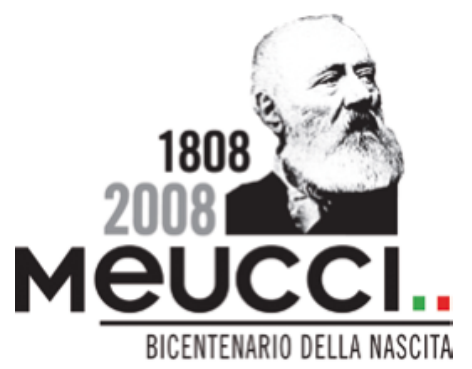

Pubblicazione promossa dal Comitato Nazionale per il bicentenario della nascita di Antonio Meucci.

(C) 2008 Firenze University Press

Università degli Studi di Firenze

Firenze University Press

Borgo Albizi, 28, 50122 Firenze, Italy

http://www.fupress.com

Printed in Italy 


\section{SOMMARIO}

Presentazione

Per la Prioritì di ANTONio Meucci nell'invenzione del telefono

Edizione anastatica

I. Notizie biografiche [i9]

II. Le PRime Rivendicazioni in Italia della scoperta Meucci [25]

III. La Stampa Americana Per la PRiorità di ANTONio Meucci [30]

IV. Il telefono Meucci alla Esposizione di Elettricitì di Filadelfia [34]

V. Il «Caveat», lo Schema di A. Meucci e lo Schema Bell [39]

VI. I Precursori del Telefono [47]

VII. Questioni di PRIORità nella INVEnZione del Telefono [53]

VIII. Trattative di Meucci con la Compagnia dei Telegrafi, con la compagnia Globe e con la Compagnia Bell [56]

IX. Cause E COMPetizioni Per le INVEnZioni telefoniche [59]

X. La Patente Bell in Italia [65]

XI. Conclusione [67]

Appendice I. Bibliografia e Documentazione [69]

Appendice II. Alcuni autografi di A. Meucci [7I] 



\section{PRESENTAZIONE}

Nell'Aprile del 2008 ricorre il bicentenario della nascita di Antonio Meucci (Firenze, 13 Aprile 1808 - New York, 18 Ottobre 1889), universalmente riconosciuto come l'inventore del telefono ed uno dei grandi personaggi che hanno contribuito a rappresentare creatività e scienza fiorentine. La Facoltà di Ingegneria dell'Università degli Studi di Firenze ha preso l'iniziativa di celebrare la ricorrenza chiedendo ed ottenendo dal Ministero per i Beni e le Attività Culturali la costituzione di un Comitato Nazionale.

Come si può ben comprendere si tratta di un riconoscimento che la Facoltà di Ingegneria fiorentina ha sentito il dovere di tributare ad un suo illustre concittadino, vittima di un'ingiustizia e che a ragione può essere annoverato fra gli esponenti più prestigiosi della tradizione tecnico scientifica di Firenze.

Nell'ambito delle proprie iniziative il Comitato Nazionale ripropone la ristampa, tramite la Firenze University Press, della relazione presentata da Luigi Respighi nel 1930 a Guglielmo Marconi, all'epoca Presidente del Consiglio Nazionale delle Ricerche, "Sulla priorità di Antonio Meucci nell'invenzione del telefono» che rappresenta la prima, autorevole, rivendicazione della priorità di Antonio Meucci di un'invenzione che ha cambiato il mondo.

Solo l'11 Giugno 2002, ad oltre un secolo dalla battaglia legale intrapresa contro Alexander Graham Bell, il Congresso degli Stati Uniti d'America, approvando la risoluzione n. 269, riconosceva finalmente che «the live and the achievements of Antonio Meucci should be recognized, and his work in the invention of the telephone should be acknowledged».

Franco Angotti

Presidente del Comitato Nazionale 



\section{PER LA PRIORITÀ DI ANTONIO MEUCCI NELL'INVENZIONE DEL TELEFONO}

Edizione anastatica 



\title{
ING. LUIGI RESPIGHI
}

\section{Per la priorità di Antonio Meuccí}

\author{
nell' invenzione del telefono
}

ROMA

a eura del Consigltó Nazionale delle Ricerche 1930-VIII 


\author{
ING. LUIGI RESPIGHI
}

\title{
Per la priorità di Antonio Meucci
}

\author{
nell' invenzione del telefono
}

ROMA

a cura del Consiglio Nazionale delle Ricerche 1930-VIII 
Stab. TIP. R. Garront - roma, Via Francesco De Sanctis, 9. 


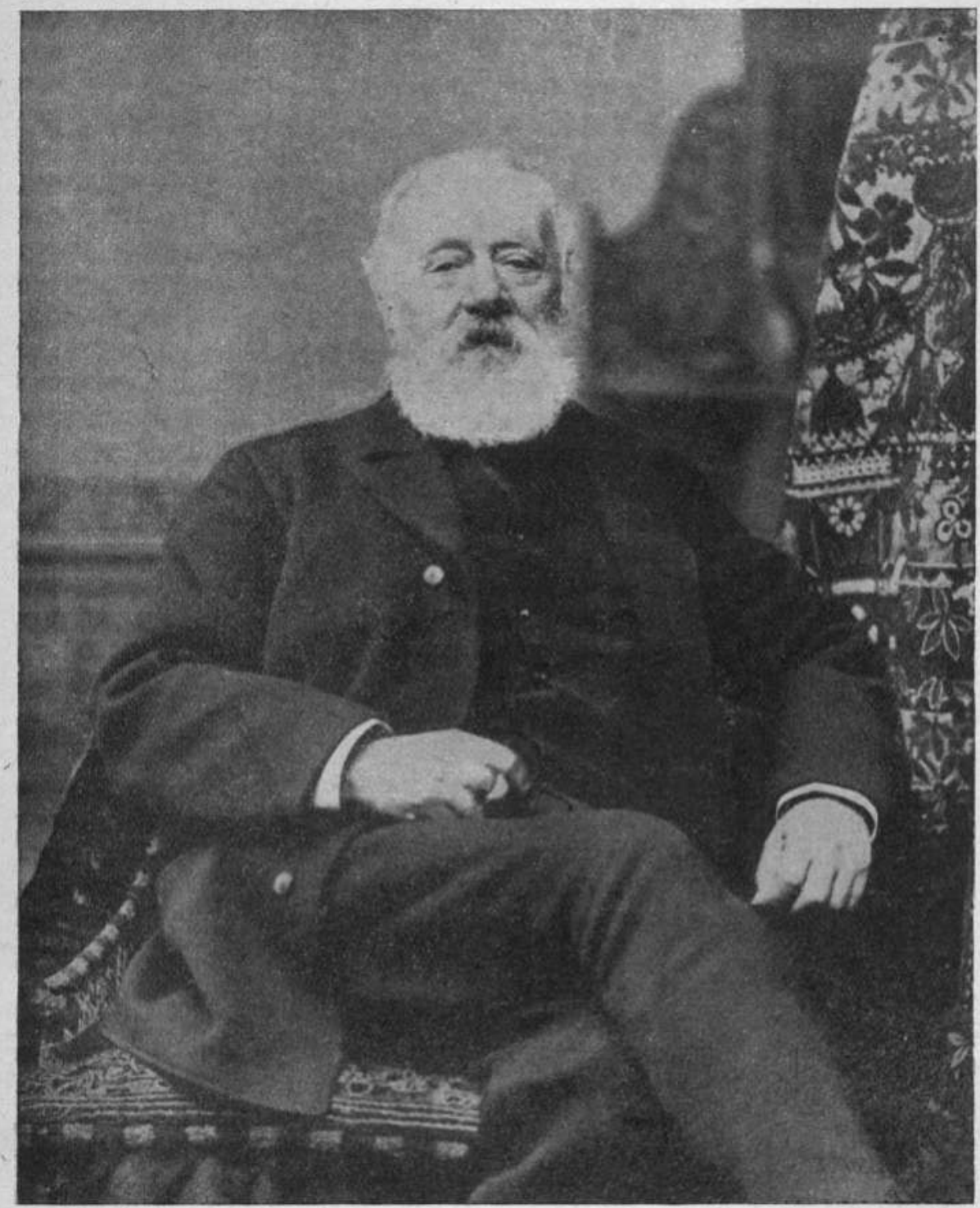

Ritratto di ANTONIO MEUCCI (Dal Corriere d'America).

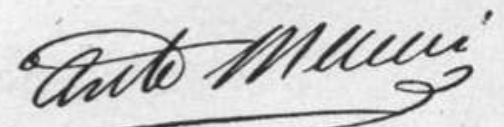




\section{IN D ICE}

I. - Notizie biografiche . . . . . . . . . . . Pag. 7

II. - Le prime rivendicazioni in Italia della scoperta Meucci . . . $\mathrm{I}_{3}$

III. - La Stampa americana per la priorità di Antonio Meucci . . . " I8

IV. - Il telefono Meucci alla Esposizione di Elettricità di Filadelfia . " 22

V. - Il «Caveat », lo Schema di A. Meucci e lo Schema Bell . . " 27

VI. - I Precursori del Telefono . . . . . . . . . . . " 35

VII. - Questioni di priorità nella invenzione del Telefono . . . . > $4 \mathrm{I}$

VIII. - Trattative di Meucci con la Compagnia dei Telegrafi, con la

Compagnia Globe e con la Compagnia Bell . . . . . " 44

IX. - Cause e competizioni per le invenzioni telefoniche . . . . \$ 47

X. - La Patente Bell in Italia . . . . . . . . . . . , 53

XI. - Conclusione . . . . . . . . . . . . . . . $\$ 55$

APPENDICE I. - Bibliografia e Documentazione . . . . . . . " 57

APPENDICE II. - Alcuni autografi di A. Meucci . . . . . . . " 59 
I.

\section{NOTIZIE BIOGRAFICHE}

Poco si conosce della vita di Antonio Meucci. Si cominciò a parlare di lui nella Stampa Americana dal $188_{3}$ in poi, in occasione delle liti e delle contestazioni circa i varii brevetti del telefono e circa la priorità della invenzione, e poi in occasione della sua morte (1889). Luigi Barzini pubblicò nel 1926 nel Corriere d'America quanto con lunghe e minuziose ricerche potè raccogliere in proposito, specialmente nei giornali Americani. Umberto Bianchi, coadiuvato dal Prof. Angelo Banti, in una memoria su A. Meucci, pubblicata nel 1923, raccolse altre notizie desunte da lettere ed appunti conservati dal fratello del Meucci, Giuseppe, deceduto nell'Ospedale di S. M. Nuova in Firenze. Anche Giuseppe Garibaldi nelle sue « Memorie autobiografiche $\gg$ ricorda la sua convivenza in America, come collaboratore ed ospite del Meucci.

Riassumiamo brevemente dalle fonti succitate le seguenti notizie biografiche.

Meucci Antonio, Sante, Giuseppe nacque in Firenze il ${ }_{13}$ Aprile 1808 , da Giuseppe Meucci e Domenica Pepe, e fu battezzato il giorno successivo nel Battistero di S. Giovanni.

Giovanissimo, fu dapprima al servizio del Granduca di Toscana Leopoldo II, addetto alla . Custodia delle porte » della città, cioè alle verifiche dei passaporti. Divenne poi meccanico attrezzista al Teatro della Pergola con 1'Impresa Lanari, ove ebbe occasione di conoscere e sposare Ester Monti, addetta alla Sartoria del Teatro.

Nel I 835 , all'Avana, scritturato come attrezzista e meccanico dalla « Tacon Opera House, usando il primitivo portavoce a cordoncino teso tra due imbuti su membrana di pergamena, per dare ordini ai macchinisti del teatro, concepi l'idea di trasmettere a distanza le vibrazioni della membrana di questo cornetto acustico, mediante la corrente elettrica, cioè di «telegrafare la parola ».

Tra il 1844 e il 1848 tornó in Italia per partecipare alla rivoluzione, raggiungendo il grado di Tenente.

Ritornato nell'Isola di Cuba, lavorava anche come elettricista in galvanoplastica, e curava i malati con l'elettricità. $\mathrm{Nel} 1849$, durante una di queste esperienze, credè di udire attraverso $\mathrm{i}$ fili, delle voci, provenienti da un paziente che si trovava alla distanza di 25 metri, e notò che usando un cone di carta, i suoni 


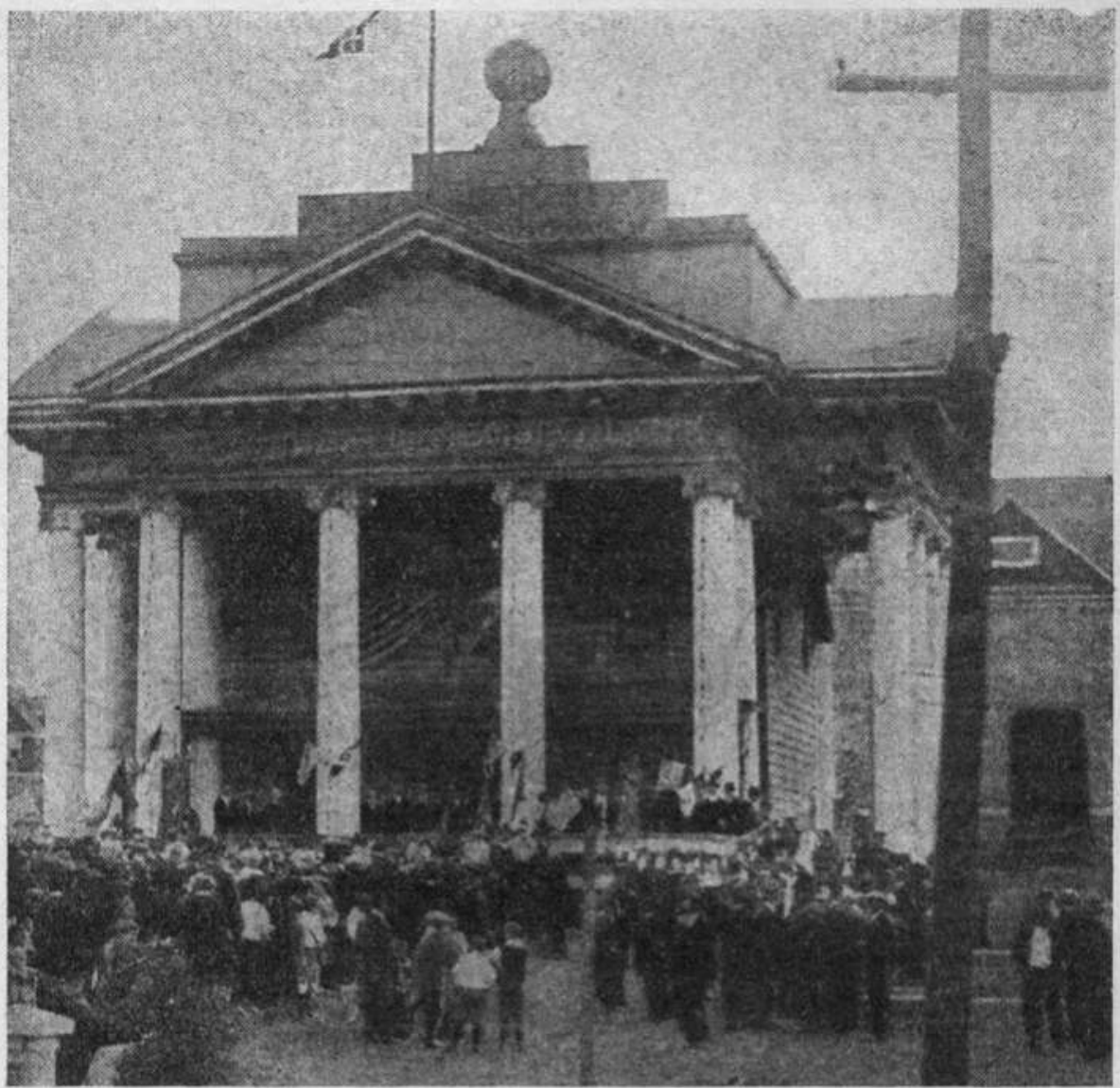

Casa e Tempio di Meucci a Clifton (Staten Island).

(Dal Corriere d'America).

aumentavano. Da ciò intuí la trasformazione della voce in vibrazioni e correnti telefoniche, e fu quindi indotto a perfezionare ed estendere le sue esperienze.

Verso il 1850 il Meucci in seguito ad un incendio che distrusse il Teatro della Tacon Opera House, dovè trasferirsi a New-York in cerca di occupazione stabilendosi a Clifton, Staten Island, isola dello Stato di Nuova York, contea di Richmond, dove acquistò un « cottage, ed impiantò una fabbrica di candele di paraffina. 
Quivi dal 1851 al 1853 ebbe ospite e collaboratore Giuseppe Garibaldi.

E interessante riprodurre testualmente dalle * Memorie Autobiografiche , la narrazione che Garibaldi fa della comunanza di vita e di lavoro con Antonio Met:cei.

"Qualche cosa bisognava fare. Un mio amico, Antonio Meucci, fiorentino e brav'uomo, si decide a stabilire una fabbrica di candele e mi offre di aiutarlo nello stabilimento.

- Detto fatto, interessarmi della speculazione non lo potevo per ragioni di m ancare i fondi; mi adattai quindi al lavoro per la condizione di fare quanto potevo.

- Lavorai alcuni mesi con Meucci che, benchè lavorante suo, mi trattò come della famiglia, e con molta amorevolezza.

* Un giorno, però, stanco di far candele e, spinto forse da irrequietezza naturale ed abituale, uscii di casa col proposito di mutar mestiere. . . . . Non mi volevano.

Tornai al lavoro del sego. Fortuna che io non avevo palesato la mia risoluzione all'eccellente Meucci e quindi, concentrato in me stesso, il dispetto fu minore. Devo confessare di più; non essere il contegno del mio principale verso di me che mi a vesse obbligato alla mia intempestiva risoluzione. Egli mi era prodigo di benevolenza e di amicizia siccome lo era la Signora Ester di lui sposa.

« La mia condizione non era, dunque, deplorabile in casa di Meucci e fu p roprio un eccesso di malinconia che mi aveva spinto ad allontanarmi da quella casa. In essa io era liberissimo: potevo lavorare, se mi piaceva, e preferivo naturalmente il lavoro utile a qualunque altra occupazione; potevo andare a caccia q ualche volta, e spesso si andava anche a pesca con lo stesso principale e con vari altri amici di Staten Island di New York che spesso mi favorivano con le loro visite.

« In casa, poi, non v'era lusso, ma nulla mancava delle principali necessità della vita sia per l'alloggio che per il vitto s.

Per il ristagno del commercio delle candele, la fabbrica dovette essere chiusa: il Meucci tentò altre industrie; una manifattura di carta d'alga a Porth Amboy, u na fabbrica di birra a Clifton, una vernice per la conservazione del legno e poi ancora una fabbrica di pianoforti, ma nessuno di questi tentativi gli riusci finanziariamente.

Appassionato per la fisica e la meccanica, aveva anche ideato e costruito un igrometro dei tipi primitivi consistente nella figurina di un Gambrinus che secondo il variare del tempo sollevava od abbassava una coppa. Prese anche ile brevetto di un e Acid Candle , che vendè per poche migliaia di dollari.

In una lettera del 14 novembre 1855 il Meucci così scriveva, sconfortato, al fratello Giuseppe, degente all'ospedale di S. Maria Nuova in Firenze:

- Se di più non vi scrivo, lo faccio per non darvi maggiori dolori di quelli che avete, perchè non posso dirvi altro che mi trovo in molto cattive circostanze di dover forse fuggire e andarmene altrove. Ho finito tutto quello che tenevo e 
non mi resta altro che la casa e terreno e fabbrica ove si fabbricavano le candele. Ma di queste ora è inutile parlarne, non si trovano da vendere. Ora mi sono messo a fabbricare pianoforti, ma anche questo mi va male.

Dunque si vede che per me non v'è fortuna e tutto quello che intraprendo non $\mathrm{mi}$ va bene. Credete a me, nel grado in cui sono preferirei di essere in patra in mezzo alla miseria ed al colera piuttosto che stare qui. . . . . . . . .

Ed infatti nel r865 la proprietà fu venduta dallo Sceriffo ad un birraio.

Il Meucci frattanto continuava le sue esperienze e nel 1856 impiantava un telefono nella sua casa, dalla camera della moglie, che era malata, alla cucina, e poi con la fabbrica di birra. Si trattava di un magnete imperfetto, con un rocchetto e diaframma chiuso in una scatola di legno e carta.

Secondo la sua testimonianza avanti alla « Circuit Court» degli Stati Uniti, egli aveva perfezionato 1' invenzione. Nello stesso anno incaricava 1' amico Enrico Bandelari che si recava in Europa, di trovare persone competenti che si interessassero della invenzione, e procurassero i capitali occorrenti, ma a nulla potè approdare, e quando tentò di istituire una « Teletrofono Company 》, (così egli chiamava la sua invenzione) non trovò che 20 dollari di capitale.

Il 28 dicembre $x 87 x$ il Meucci presentò all'Ufficio delle Patenti degli Stati Uniti la domanda ufficiale di brevetto, il cosiddetto e caveat ", ossia una richiesta o diffida provvisoria di garanzia preventiva, per potere poi completare e dettagliare, nelle forme volute, la domanda definitiva di brevetto.

Le condizioni economiche del Meucci erano frattanto peggiorate, anche per il fatto che, trovatosi nel ${ }_{1} 872$ nella esplosione del ferry-boat Westfield, ne restò infortunato ed invalido per parechi mesi. In tali contingenze la moglie dovette impegnare e vendere tutto, compresi i rudimentali apparecchi telefonici che per 6 dollari andarono a finire in mano ad un rivenditore, certo Sig. Fleming di Clifton. In conseguenza di tale stato di preoccupazioni e di effettiva povertà, per la quale la Contea gli dovette passare un assegno di 25 dollari, il Meucci non ebbe nè tempo nè mezzi per completare e presentare la domanda definitiva del brevetto.

Si giunse cosi al 14 febbraio 1876 quando, con intervallo di 2 ore apparvero due domande di brevetto, presentate dal Sig. Patrik a nome del Prof. Alessandro Graham Bell e dal Sig. Jonathan a nome di Elisha Gray di Chicago, aventi appunto per oggetto la invenzione del telefono: ma la domanda del brevetto Meucci, anteriore ad entrambe, era purtroppo oramai decaduta!

Tratteremo qui appresso più dettagliatamente quanto delle avverse vicende della attività del Meucci riguarda la parte telefonica propriamente detta, e cioè le sue infruttuose trattative con la New-York Districht Telegraph $\mathrm{C}^{0}\left({ }_{1872}\right)$ e con la Bell Telephone $\mathrm{C}^{0}\left(\mathrm{r}_{88}\right)$ - il tentativo di applicare il telefono con la Globe Telephone $\mathrm{C}^{0}$ di Filadelfia ( 1884 ) - le varie cause contro la Bell per l'annullamento del brevetto e questioni di precedenza di altri presunti inventori dal $188_{3}$ in poi. 
Il Meucci si trovava ormai da tempo, come abbiamo veduto, in cattive condizioni finanziarie, anzi in povertà addirittura; ed anche quando nel $1884-85$ si uni alla Globe Tel. $\mathrm{C}^{0}$, non ne ricavò che una « royalty » di ${ }_{15} 0$ dollari al mese.

$\mathrm{Si}$ disse, e venne anche riportato da alcuni giornali, che in relazione alle note vertenze giudiziarie della Globe contro la Bell, il Meucci avesse percepito a titolo di transazione roo.00o dollari.

Ciò peraltro, non solo non risulta effettivamente avvenuto, ma è smentito da quanto il Meucci scriveva in quel tempo al fratello.

« Non mi piace di dar nuove cattive; così mi limito di scrivere a nessuno; sai e sanno tutti bene quanto ho lavorato e quanto lavoro ancora, ma adesso non mi manca che lavorare la strada che conduce all'eternità. . . . . . . . . .

Tutto ho perduto e vivo per limosina, questo è quanto vi posso dire e no n di più per non vi rattristare. Vorrei essere costà per riposare le ossa mie ».

Ed ancora il 12 giugno 1889 , quando si aveva speranza che $i$ tribunali gli dessero ragione nella priorità della invenzione del telefono, pochi mesi prima di morire egli scriveva all'amico Montelatici di Firenze. \& Io ho la voce, altri la noce. Comunque sia, spetta la definitiva decisione alla Corte d'Appello s.

Non ebbe tale soddisfazione il povero Meucci, e, mentre la Compagnia Bell sviluppava le sue immense reti di impianti e di affari, egli invece soffriva la mi seria, solo sorretto da un piccolo assegno mensile accordatogli dal Governo degli S. U., come per gli indigenti, e del quale non potè fruire che per pochi mesi.

Già malato da qualche tempo e rimasto vedovo, si aggravò nel mese di ottobre 1889 e il giorno 18 dello stesso mese all'età di 81 anni e mezzo, morì nella casa P. O. Boz 195 a Clifton di Staten Island dove aveva passato tanta parte della sua travagliata esistenza. Vaneggiando negli ultimi momenti di vita parlava ancora di telefono, di speranze e di invenzioni.

Ebbe onoranze solenni dalla colonia italiana, e dagli americani stessi che 10 amavano e veneravano. La sua casetta, che ospitò Garibaldi, venduta, come dicemmo, ad un birraio del luogo, venne donata generosamente dal proprietario alla Colonia Italiana, e fu poi racchiusa, come in un monumento, da un artistico tempio architettonico che abbiamo riprodotto qui innanzi a pag. 8 .

Ma, Lui morto, la sorte si mantenne avversa agli eredi. Il fratello Giuseppe riprese le pratiche per ottenere almeno una riparazione dalla Compagnia Bell, affidandole agli stessi due esecutori testamentari nominati dal Meucci e residenti a New-York. Si interessarono della cosa il Comm. Damiani, 1' On. Luciani, ed altre personalità, e lo stesso Ministero degli Esteri del tempo, ma a nulla poterono approdare, pur non essendovi dubbio che l'invenzione del Telefono spettasse al Meucci.

Anche sul valore degli oggetti rimastigli, segaatamente sui cimeli di Giuseppe Garibaldi, il Meucci si era illuso. Nelle disposizioni testamentarie aveva 
lasciato per riconoscenza, un dono di 100 dollari a ciascuna delle due figliuolette dell'amico Nisini, che aveva avuto molte cure per lui negli ultimi anni di vita; invece la vendita all'asta pubblica degli effetti personali e dei mobili non rese che I9I dollari, insufficienti a coprire le spese: e il lettuccio dove aveva riposato Garibaldi, fu venduto appena a valore di ferro vecchio, per sette franchi!

(Vedere in appendice altre lettere di Meucci) 
II.

\section{LE PRIME RIVENDICAZIONI IN ITALIA DELLA SCOPERTA MEUCCI}

Emigrato in America ancora venticinquenne, Antonio Meucci studiò, espe rimentò, e perfezionò colà il proprio apparecchio e tentò di portarlo a pratiche ed industriali applicazioni, Perciò in Italia nessuno conobbe ed ebbe ad interessarsi in principio di tale invenzione, né è possibile rintracciare in Italia campioni degli apparecchi e degli oggetti che vennero ideati, costruiti ed esperimentati dal Meucci. Anzi un tentativo di applicazioni dell' apparecchio stesso, fatto in Italia dal suo amico Bandelari nel 1860 , non ebbe alcuna riuscita.

Viceversa le prime esperienze che si fecero in Europa ed in Italia verso il I880 apparivano già sotto il nome di * Telephon Bell , ed i primissimi impianti di reti telefoniche nel 1881 a Genova, Torino, Milano, poi a Firenze e via via in tutte le altre città avvennero per iniziativa e sotto il controllo e cointeressenza della = International Bell Telephone C. s (Ltd) di New York, della Italo Americana Bell, e della Compagnia Bell di Anversa, titolari del brevetto in Europa, ed associate alle Società Italiana Ligure, Italia Centrale, Romana e Generale Italian a nell' impianto ed esercizio dei Telefoni in Italia.

Nessuno dunque in Italia aveva prima pensato a rivendicare ad Antonio Meucci il merito dell'invenzione del telefono. Ed invero in uno dei primissimi trattati di telefonia edito a Milano da Ulrico Hoepli nel r88o (II Telefono nelle sue origini e nelle sue applicazioni) 1' autore D. Piccoli, sviluppando cronologicamente le prime fasi della scoperta del Telefono e descrivendo ed illustrando detta gliatamente i vari sistemi ed apparecchi dei primi inventori, non fa tuttavia alcun cenno di A. Meucci.

Nella stampa americana ed inglese si cominciò a parlare del Meucci verso il $188_{3}$ e $188_{4}$ in occasione della Esposizione di Filadelfia e delle competizioni di brevetti tra le American Bell, la Globe Telephon C., il Meucci e vari altri presunti inventori.

Nel fascicolo di settembre e ottobre 1884 la Rivista italiana Il Telegrafista, diretta dal compianto Ispettore dei Telegrafi Comm. Fedele Cardarelli, riproduceva la documentazione che qui appresso esporremo, in appoggio alla priorità della scoperta del Meucci. 
Il dott. Italo Brunelli in una conferenza tenuta presso la R. Scuola d'Applicazione degl Ingegneri in Roma il 6 maggio 1900 descriveva l'invenzione del Meucci, mettendone in evidenza la priorità.

Il 17 giugno Igor, inaugurandosi la rete telefonica della Toscana, la Società Telefonica dell' Italia Centrale presentava al Ministro del tempo On. Pascolato, nel palazzo Grocco a Firenze, un artistico busto di A. Meucci.

Il I2 ottobre rgor nella riunione annuale effettuatasi in Roma, della Associazione Elettrotecnica Italiana, il Prof. Angelo Banti tenne una conferenza su Antonio Meucci corredata di dati e notizie del massimo interesse e concludeva col proporre la nomina di una Commissione per completare le indagini, le ricerche e gli studi relativi onde rivendicare al Genio Italiano l'invenzione del telefono, ma la cosa non ebbe seguito.

Nella seduta del 29 gennaio I903, discutendosi alla Camera Italiana il progetto di Legge sul Servizio Telefonico (Testo. Unico tuttora vigente) il Deputato On. Rava rivolgeva un memore omaggio al Meucci nei termini seguenti:

........ s e credo oggi bene ricordare qui, oltre il nome del giovane Marconi, - che è tanto alto ora nella scienza italiana e che sarà opportunamente onorato - con l'ordine del giorno dell'On. Frascara Giacinto, anche il nome di un povero - italiano dimenticato, quell'Antonio Meucci, esule fiorentino e fido amico e com- pagno di Garibaldi, e ricordato con affetto nelle memorie dell'eroe, che fu ospite - suo in giorni tristi, che a New York, fin dal 1850 , studiando e provando, inventò - il telefono e gli diede il nome... *

A sua volta 1'On. Frascara, illustrando un ordine del giorno sul medesimo Disegno di Legge e inviando un voto di plauso e di riconoscenza a Guglielmo Marconi, si esprimeva come segue:

* mi pare che nella discussione di questa Legge la nostra mente debba risa-

* lire alla gloria di quei grandi italiani che seppero strappare segreti di così

* vitale importanza alla natura, cominciando da Volta, che primo fece scaturire

- la favilla che si gran fiamma ha secondato, e venendo a Pacinotti e a Galileo

- Ferraris, ai quali è dovuto il sistema di trasportare forze ingenti sempre mag-

* giori a distanze meravigliose; a quell'insigne italiano, quasi sconosciuto, che

* fu nominato dal nostro collega Rava, cui pure è dovuta l'invenzione del tele-

* fono, l'italiano Meucci che fu amico di Garibaldi e fu il Nestore della Colonia

- Italiana in America, verso la metà del secolo scorso, e finalmente al nome di

* Guglielmo Marconi, il quale irradiò di nuova luce la scienza elettrica, e che ben * si può dire rappresenti il genio moderno dell'Italia, l'anelito affannoso di luce * e di verità scientifica, che domina lo spirito delle nazioni più colte dell' età * nostra...... *

Poco dopo, onorandosi al Consiglio Comunale di Firenze Guglielmo Marconi, il Consigliere Bombicci rievocando il nome di Meucci proponeva che venisse 
apposta in suo onore una lapide nella casa dove egli nacque; ma la proposta, presa in considerazione, non ebbe poi seguito.

Nel Igo6 la Società Telefonica dell'Alta Italia presentava nel frontespizio dell'elenco degli abbonati di Milano, una bella fotografia di A. Meucci, quale inventore del telefono.

Nel Trattato di Telefonia edito nel 1906, gli autori Brunelli e Longo, passando in rassegna vari precursori del telefono e descrivendo i sistemi, rivendicavano al Meucci la priorità della invenzione.

La Rivista Telegrafi e Telefoni (nel fascicolo dell'Agosto 1922) associandosi alle onoranze che si rendevano in America al Prof. Alessandro Graham Bell in occasione della sua morte (2 agosto 1922 ), pur riconoscendo $i$ grandi meriti dell'estinto, metteva del pari in evidenza anche il posto d'onore spettante al Meucci, che inventore del Telefono fin dal 1849 , aveva preso il brevetto agli Stati Uniti solo nel I 871 e nel 1872 aveva presentato ad una Compagnia Americana di Telegrafia la sua invenzione perchè venisse sperimentata.

L'On. Umberto Bianchi nel marzo 1923 rivolgeva a S. E. il Presidente del Consiglio On. Mussolini la seguente interrogazione con domanda di risposta scritta:

* Se non trovi opportuno decretare una inchiesta ufficiale che rivendichi

- finalmente al grande italiano Antonio Meucci la priorità della invenzione del

* telefono contro la invalsa credenza della opinione pubblica mondiale che l'at-

- tribuisce all'americano Bell. .

S. E. Mussolini, con nota del 4 maggio successivo, rispondeva nei termini seguenti:

* Come tutte le grandi invenzioni, destinate a lasciare di sè traccia indelebile * nella storia della civiltà umana, anche il telefono ebbe i suoi precursori, il genio * divinatore della importanza e della possibile realizzazione pratica della scoperta, * ed infine il fortunato ingegnoso realizzatore degli apparecchi destinati a diffon* dere fra gli uomini la celebrità del suo nome, insieme con la novità e la utilità

* del nuovo mezzo di comunicazione.

* Ma il primo apparecchio capace di trasmettere la parola fu esposto al pub* blico da Alessandro Graham Bell, nato ad Edimburgo (Scozia) nel 1847, ed « emigrato in America nel 1870 . Si spiega quindi come il nome di Bell venisse - così indissolubilmente legato a quello del telefono, dopo che nel r876 il suo

- apparecchio fece la prima apparizione all'esposizione di Filadelfia, e ricono-

* sciuta al Bell la priorità della invenzione contro l'americano Elisha Gray, che - la reclamava per se.

* Strana coincidenza, la quale fu potuta solo in seguito spiegare, quando si - trovó che il vero inventore del telefono non era nè Bell, nè Gray, ma l'italiano " Antonio Meucci, nato a Firenze il 1808 ed emigrato in America nell' età di 25 * anni, e quando vennero alla luce degli interessanti particolari della vita di 


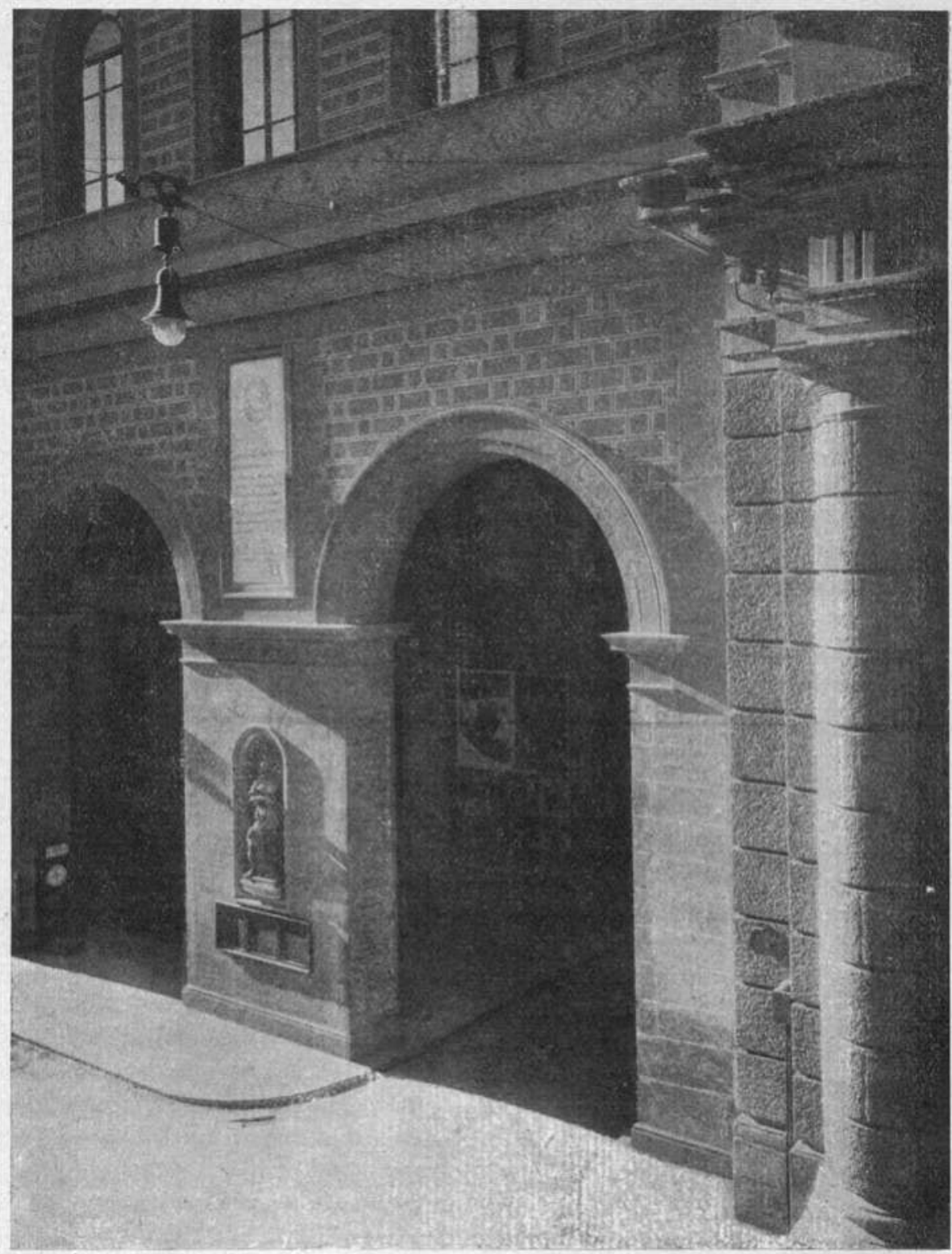

Monumento ad A. Meucci nel Palazzo delle PP. e TT. a Firenze. 
« quest'ultimo, che rivelarono come eglı versasse in grande miseria e come la - moglie vendesse, per ritrarne sostentamento, molti degli apparecchi che servi* vano agli esperimenti del marito.

* La rivendicazione della priorità e della gloria del Meucci incominciò nel - $\mathrm{I} 884$, col processo intentato dalla Globe Téléphone Company per opporsi all'in« vadente monopolio della Compagnia Bell proprietaria del relativo brevetto; * risultò allora che il Meucci aveva fatta la scoperta fin dal r 849 in Avana; che * poi, negli Stati Uniti, egli aveva nel 1871 , registrata la domanda di brevetto. * rinnovando la registrazione finchè non gliene mancarono $\mathrm{i}$ mezzi. *

Nel 1923 dovendosi istituire ad iniziativa della Siti di Milano, una Compagnia per impiantare ed esercitare servizi telefonici, si pensò a fregiarla del nome di Antonio Meucci. L'On. Umberto Bianchi, conterraneo del Meucci, fu invitato, nell'occasione, a riprendere e sviluppare la sua iniziativa, di rivendicare cioè al Meucci la gloria dell'invenzione del Telefono. Il Bianchi, con la collaborazione del Prof. Banti e di competenti è volonterosi cittadini, pubblicò una speciale monografia su Antonio Meucci, ricordandone con nuove notizie e documentazioni inedite tutti i particolari della vita, la storia della scoperta del telefono, le vicende e traversie del glorioso e tuttavia sfortunato inventore.

Il I5 giugno 1924, inaugurandosi a Firenze il Palazzo delle Poste e Telegrafi, venne ivi apposta una lapide in onore di Antonio Meucci, e l'On. Lupi in rappresentanza del Governo Nazionale, e alla presenza di autorità civili, militari e politiche e di folto attentissimo púbblico, commemorò solennemente con un pode. roso discorso il grande inventore.

Sotto al medaglione in cui è riprodotta l'effigie venne incisa una iscrizione che il * New York Times \$ (22 novembre r925) volle sarcasticamentc commentare col conseguente richiamo in appoggio alla priorità Graham Bell nella invenzione del telefono. Luigi Barzini, direttore del c Corriere d'America » di New York, senti il dovere dell'amor proprio nazionale di insorgere, col suo giornale, in difesa del Meucci e di riprendere la trattazione a fondo, ancor più di quanto era stato fatto fino allora, della famosa questione della priorità della scoperta del telefono.

Nei numeri del 7; $14 ; 2$ gennaio, 8 febbraio, 7 e 14 marzo 1926 infatti è raccolta e sviluppata una tale serie di notizie, dati e documenti da giungere alla assoluta ed indiscutibile conclusione che effettivamente il merito e l'onore della scoperta del telefono spettano all'Italiano Meucci.

Lo stesso * New York Times» in occasione della morte del Meucci, come vedremo, era stato un sostenitore della priorità di lui nell'invenzione; e chi oggi avesse ancora qualche dubbio, in proposito, leggendo le esaurienti e poderose esposizioni suaccennate, ne riporterebbe la convinzione assoluta e nelle fortunose circostanze della vita e nelle vicissitudini della invenzione del Meucci, troverebbe ancora più grande la figura di lui. 
III.

\section{LA STAMPA AMERICANA PER LA PRIORITÀ DI ANTONIO MEUCCI}

\section{Dal New York Times (19 Ottobre 1889) in occasione della morte di MEUCCI.}

- Egli (Meucci) era nativo di Firenze, dove vide la luce ottantadue anni fa. Ivi studiò preparandosi alla carriera teatrale. Il suo genio per la meccanica, però, interferi con la carriera scelta, e alla fine egli divenne macchinista teatrale. I suoi studi lo condussero a specializzarsi nella elettricità e nel ${ } 835$ andò all'Avana dove diventò elettricista in galvanoplastica. Ritornò in Italia in tempo per partecipare alla rivoluzione del $1844-48$, conquistandosi il grado di tenente. Per evitare di essere catturato dagli austriaci, tornò all'Avana. Poi, con un capitale di circa 20.000 dollari, venne in questo paese, e nel 1850 fu preminente fra quelli italiani che accolsero Garibaldi messo al bando. Egli era stato membro della stessa associazione rivoluzionaria alla quale aveva appartenuto Garibaldi, e sorse fra $i$ due una strettissima amicizia, che si estese agli affari. Tutti e due andarono a domiciliarsi a Clifton, dove Meucci eresse una fabbrica di candele di paraffina Egli comprò un « cottage » dove vivevano, lul, sua moglie e Garibaldi. Quest'ultimo rímase con loro dal 1851 al 1853 .

- Ma Meucci non era soddisfatto di questa impresa. Cominciò la manifattura di carta d'alga a Perth Amboy, e poi promosse una fabbrica di birra a Clifton. Ma egli non era un buon amministratore, e la sua impresa falli. Nel r865 la sua proprietà fu venduta dallo Sceriffo. Da quel tempo fino alla morte egli è rimasto in povertà.

- Fin dal 1856 , Meucci fece un telefono (dieci anni prima di Bell; N. d. r.) che si estendeva dalla camera di sua moglie ammalata alla cucina, e la sua camera era connessa con lo stesso sistema alla fabbrica di birra. Questo telefono era composto di un magnete imperfetto con un rocchetto e un diaframma ed era chiuso in una scatola di legno e di carta. Nel 1860 , secondo la sua testimonianza avanti alla «Circuit Court » degli Stati Uniti, egli aveva perfezionato la sua invenzione. La sua scoperta del principio del telefono è alquanto interessante. Quando era all'Avana egli curava dei malati con l'elettricità nella sua casa, una volta egli credè di udire delle voci attraverso dei fili elettrici, e, investigando, 
trovò che il suono veniva dalle labbra di un paziente che era lontano ottanta p iedi. Usando un cono di carta scoprì che il suono aumentava di volume. Questo avveniva nel $184 \mathrm{I}$ (trentacinque anni prima che Bell domandasse le sue patenti. N. d. r.). Egli chiamò la sua invenzíone il a teletrofono s, e tentò di introdurlo in Italia, ma non riusci. Continuò a lavorare sopra a questo fino al 1864 , quando gli parve di averlo perfezionato. Ma allora, egli quasi perdette la vita nell'esplosione della caldaia del * ferryboat Westfield, e per cinque mesi fu incapacitato Sua moglie impegnò tutto quanto essa possedeva nel mondo, ed egli vendette il suo telefono ad un mercante di roba vecchia per 6 dollari. Meucci guari, e nel 187 I organizzò una = Teletrofono Company s, ma non trovò che 20 dollari di capitale.

- Egli registrò « Caveat * (dichiarazioni di invenzione che si depositano come primo atto per ottenere patenti) nel 1869 , 1870 e 1871 , annunziando la sua invenzione per assicurarsi le patenti e cercò un appoggio finanziario. Ma rimase avvilito quando, essendo eccessivamente povero, nel 1876 lesse che Bell sí era assicurato patenti per lo strumento di cui egli dichiarava 1 'invenzione. In seguito egli fu preso nella \& Globe Telephone Company », e quando Bell ebbe causa con la a Globe », Meucci vendè a quest'ultima le sue patenti per poche migliaia di dollari, che i creditori si presero ».

\section{Dal World (19 Ottobre 1889).}

- L' invenzione del telefono risale al tempo in cui Meucci risiedeva a Cuba, nel r849. Egli era allora direttore dei meccanici della = Tacon Opera House *, e dedicava molto del suo tempo in esperimenti di elettricità. Mentre studiava una teoria per la cura delle malattie per mezzo dell'elettricità, egli mise insieme un filo conduttore e una batteria per formare un circuito completo. Ogni filo finiva connesso ad una piastra di rame. Due uomini erano in due diverse camere: Meucci ed uno dei suoi amici. Ciascuno di loro pose la piastra in bocca. La scossa fece mandare all'amico una esclamazione. Meucci, all'altro capo del filo teneva la piastra fra i denti, e udì la voce. Continuò a esperimentare questa scoperta. Nel maggio del 1851 perfezionò un cono vibratorio di carta, e conversava debolmente con altra gente attraverso alla strada. Quando Meucci venne a New York, vi fu una grande lotta fra la sua povertà e il suo desiderio struggente di avanzare la sua invenzione. Per un anno o due ricevette una * royalty * di $\mathrm{r}_{50}$ dollari al mese dalla * Globe Telephone Company s di Filadelfia...

- Una quantità di invenzioni minori furono fatte da questo scopritore del telefono. Fra tante era un barometro, (Sic) considerato una grande novità ai suoi giorni. Consisteva nella figura di un Gambrinus che levava la coppa piena di 
birra, e la abbassava all'avvicinarsi di una tempesta. Egli inventò pure un'acid candle, le cui patenti vendè per 10.000 dollari.

- A proposito, fu al tempo in cui Meucci fabbricava candele, che ebbe fra i suoi impiegati Giovanni Morosini, che è adesso il ricco banchiere ben conosciuto .

\section{Dichiarazioni dell' Avv. A. P. ULMAN.}

Una personalità nota e stimata, l'Avv. A. P. Ulman, che era stato intimo amico del Meucci, viveva nel 1926 a Staten Island, mentre il \& Corriere n aveva ripreso la campagna di rivendicazione in favore dell'inventore italiano.

Lo Staten Islander, del 6 Gennaio 1926, pubblicava le seguenti concrete dichiarazioni :

* Che Antonio Meucci, un residente di Rosebank e amico del patriota italiano Generale Garibaldi, inventò il telefono diciassette anni prima di quando Alessandro Graham Bell dichiarasse di aver fatto la scoperta, è affermato da A. P. Ulman, Avvocato di Staten Island, vivente nella Huguenot Av. N. 638 ed esercitante in Manhattan.

- Mr. Ulman, che è nato in Staten Island da discendenza ugonotta, conobbe Mr. Meucci personalmente, e, in una dichiarazione giurata afferma essere il solo testimonio oculare vivente della priorità dell' invenzione dello scienziato italiano.

Ecco il racconto di Mr. Ulman :

« Io nacqui in Tompkinsville Staten Island nello Stato di New York; il 20 Maggio I849. Dall'anno 1850 al 1870 risiedetti a Clifton, Staten Island, a circa ro minuti di strada dalla casetta occupata da Antonio Meucci e dal Generale Garibaldi e i loro famigliari, situata in una strada chiamata Willow Avenue, vicino alla fabbrica di birra Meyer e Bachmann. Quella stessa casa, dopo la loro morte, fu trasportata alla località attuale sulla Tompkins Avenue, a poca distanza dalla sua posizione originaria $»$.

- Prima del 1858 Meucci era in ottime relazioni con mio padre Bernard Emille Ulman, e più tardi divenne intimamente amico mio.

« Nel 1858 , Antonio Meucci e il Generale Garibaldi si dedicarono alla manifattura di candele di stearina alcune delle quali, ricordo, erano lunghe cinque o sei pollici, colorate in rosso, verde, giallo, bianco, e venivano adoperate per decorare alberi di Natale, ecc.

* La loro fabbrica era situata vicino ad un approdo che si chiama \& Vanderbilt Steamboat Ferry Landig in una strada detta Amas Street e ad un cinque minuti di strada dalla mia casa. 
« Io ero allora un ragazzo di otto o nove anni e visitavo frequentemente la loro fabbrica. Cosi entrai in buone relazioni col Generale Garibaldi, che era alto e molto più sottile di Mr. Meucci. Egli portava sempre una camicia rossa nella f abbrica, aveva capelli castani, un'ampia fronte, occhi grandi, brlllanti ed intellettuali, una espressione piacevole e gentile.

- Antonio Meucci era alto circa cinque piedi e sette pollici, pesava presso a poco duecento libbre, aveva una larga testa, e degli occhi straordinariamente intelligenti, brillanti, penetranti, dolcemente espressivi. I suoi modi erano gentili, franchi, caldi e magnetici.

- Circa dall'anno 1858 , fino a quando chiusero la fabbrica di candele, quando passavano giornalmente avanti alla mia casa per andare al lavoro, li incontravo spesso e conversavo con loro. In una di quelle occasioni, all'angolo di Simenson Avenue e Bay Street presso la mia dimora, Mr. Meucci ed io avemmo una lunga conversazione molto interessante. Quello che egli mi disse, lo ricordo bene, riempi la mia mente di stupore e di interesse per molti giorni, e ancora adesso, alla mia etá, è perfettamente chiaro nella mia memoria.

- In quel momento e in quel luogo, Antonio Meucci mi disse, e pensate bene che questo avveniva diciassette anni prima del 1875, quando Alexander Graham Bell dichiarò di aver inventato il meraviglioso telefono: «Vieni a casa mia, e ti mostrerò la mia macchina parlante ». Io gli domandai : « È viva? , ed egli mi ri spose: « No ma vieni e te la mostro s. Deploro di dover dire che non andai a c asa sua a vedere tal cosa, ma ricordo quanto mi scervellai per capire come parlasse.

« Molti anni dopo avevo un ufficio legale al N. I State Street in New York, Mr. Meucci venne con un carico di documenti e disse : Ecco le copie delle dimostrazioni, e dei diagrammi che furono adoperati in Corte quando la mia invenzione mi fu portata via . Io dissi: «Come ve l'hanno portata via? »-Egli rispose: « Non ho più denaro e non posso far fronte alle spese per gli avvocati e 1a Corte: non posso continuare e lottare, mentre dall'altra parte hanno tutto il denaro per seguitare a lottare. $\mathrm{Ma}$ io sono il vero inventore $»$. Io replicai : « Non ho alcun dubbio: voi siete l' inventore del telefono, e ricordo bene quando, essendo io un ragazzo, voi $\mathrm{mi}$ incontraste presso alla mia casa a Clifton e m'invitaste a vedere la vostra macchina parlante s.

Il Baltimore Sun del 21 ottobbre I $885_{5}$ riceveva da New York il racconto della invenzione di Meucci e scriveva : \& Il caso Meucci è tale da fare fortissimo appello alle simpatie di tutti $» e$, narrando l'origine della scoperta, definiva lo strumento di Meucci come un «eccellentissimo telefono e ricordava gli sforzi di Meucci per interessare alla sua invenzione la Compagnia dei Telegrafi di New-York. 
IV.

\section{IL TELEFONO MEUCCI \\ ALL'ESPOSIZIONE UI ELETTRICITÀ DI FILADELFIA}

(Settembre 1884)

Il Maggiore W. C. Barney, personalità competentissima specialmente in materia di brevetti e delle prime scoperte telefoniche, nel n. 359 dell' 1 I ottobre 1884 dell'autorevole Rivista Inglese The Telegraphic Journal and Elettrical Review, nella sua relazione preliminare sulla Esposizione di Elettricità di Filadelfia, premette che tale Esposizione è anzitutto interessante per la storia del Telefono che vi ̀̀ completamente sviluppata, e passando in rassegna i vari espositori nei singoli campi della elettricità, si sofferma, per quanto concerne la parte telefonica, sui seguenti espositori:

American Bell Telephone Company: ha una centrale in funzione che collega 25 espositori con gli uffici della esposizione e con tutti gli abbonati della Centrale di Filadelfia. Sono presentati i modelli dell'originale apparecchio di Bell che, osserva lo scrittore, sarebbe considerato come il primo telefono, il quale parere però non è condiviso da parecchi altri. Sono pure presentati oltre i telefoni che funzionano nella mostra, anche i telefoni originali di Edison e di Blacke, e molteplici altri telefoni e trasmettitori, in circa 250 specie, costruite ed usate a scopo commerciale.

Assai più a lungo e diffusamente lo scrittore riferisce sulla ditta espositrice:

Globe Telephone Company di N. Y. - Osserva il relatore che questa compagnia, degna di particolare menzione, era stata costituita per mettere in lavorazione ed esercizio i brevetti Shaw, ed anche le invenzioni Hadden, tenute allora segrete e che perciò non era possibile di descrivere.

$\mathrm{Ma}$ il maggiore interesse, prosegue to scrittore, è dato alla Mostra dalla invenzione del Mrucci, della quale, oltre a quanto già era stato precedentemente riferito in vari altri numeri della rivista, egli dà ulteriori informazioni. Lo scrittore avverte che, pendendo una vertenza tra la Globe e la Bell, i documenti presentati sono solo una parte di quelli esibiti per la causa in questione e che ve ne sarebbero ancora altri più circostanziati sui quali lo scrittore non 
può intrattenersi per ovvie ragioni di delicatezza, dovendo la vertenza essere discussa e definita da una Corte degli Stati Uniti.

Fa quindi seguire il Dr. Barney, nel succitato fascicolo della rivista londinese il testo degli * Affidavit » (dichiarazioni giurate) a favore dell'inventore Meucci, e il * Caveat » del relativo brevetto, i quali documenti, data la loro capitale ed assoluta importanza, vengono qui appresso integralmente riprodotti.

\section{Documentazioni ed "Affidavit , in difesa di MEUCCI.}

Prosegue la succitata Relazione del Barney nella presentazione dei documenti esibiti dalla "Globe $\mathrm{C} o$, " alla Esposizione di Filadelfia a rivendicazione della priorità del Meucci.

La deposizione giurata di Axronio Meuccr, residente a Clifton, Staten Island, è diretta a stabilire che egli è il vero e primo inventore di un sistema per telegrafare per mezzo del suono e che egli ne fece la scoperta in Avana, isola di Cuba, nel 1849 . Che egli continuò gli esperimenti nel 1852.53 ecć. ed anche a Staten Island, e che nel 1860 egli diede incarico al suo amico Enrico Bandelari, residente allora a New York ed ora a Toronto, che si recava in Europa, d'interessare alla sua invenzione delle persone competenti. Che nel 1871 egli fece registrare una diffida (Caveat) nell'ufficio dei brevetti degli Stati Uniti e continuò la registrazione finchè non gliene mancarono i mezzi. Asserisce che nel 1872 in compagnia del suo amico A. Bertolino, residente a New York, cercò interessare alla cosa il Sig. Grant allora presidente della Compagnia New York District Telegraph, e fece per mezzo della stampa delle frequenti rivendicazioni del suo diritto di priorità d'invenzione, una delle quali fu ripubblicata a New York il 6 Marzo r88o. Di più afferma con giuramento che, per causa dei danni avuti nell'esplosione del ferry-boat a Westfield di Staten Island, la cattiva salute che ne fu conseguenza, la perdita di tempo, di moneta e di lavoro, gli fu assolutamente impossibile di far le spese necessarie pei modelli, disegni, onorari, legali ecc. per completare la domanda di brevetto per la sua invenzione. La deposizione porta la data del 30 Marzo I880.

La deposizione di ANGelo BERTol,INo sopramenzionato, residente nella New York City, stabilisce che nel 1871 egli ebbe a fare qualche disegno relativo alla invenzione del Meucci ed al suo sistema di trasmissione della voce umana per mezzo dei fili telegrafici, chiamato telefono. Che nel 1872 , il Meucci servendosi di lui come interprete, spiegò la propria invenzione al Sig. Grant e lo richiese di fare qualche esperimento per mezzo dei fili della sua Compagnia. Che consegnarono al Sig Grant una descrizione in inglese con figure dell'invenzione, unitamente 
ad una copia della diffida (di cui parla il Meucci nella sua deposizione). Che il Sig. Grant promise che avrebbe fatto l'esperimento alla prima opportunità : poi dà conto di vari ritardi, finchè, dopo due anni, il Meucci perdè la speranza di aiuto per parte del Sig. Grant, e, a richiesta del Meucci, si recò dal Sig. Grant per ritirare le carte consegnateli e cae questi gli disse di averle imprestate, e nè lui nè il Meucci riuscirono più a recuperarle. Egli corrobora pure la deposizione del Meucci per quanto si riferisce alla salute ed ai mezzi dell'inventore, nonchè alla esplosione del ferry-boat.

La deposizione di ESTER MEUCCI, moglie dell'inventore, dice che all'Avana, il Meucci, mentre funzionava da Direttore del meccanismo nel Teatro dell'Opera della Città, attendeva qualche volta a lavori di galvanoplastica e fece degli esperimenti per riescire a parlare ad una persona collocata a qualche distanza per mezzo "di fili attaccati alla sua batteria elettrica ». Che la persona con cui fu fatto l'esperimento, come anche lei stessa, senti le parole attraverso del filo, ma non abbastanza chiare secondo suo marito. Narra di quanto danno furono al proprio marito i suoi reumatismi incurabili, e corrobora le asserzioni del Sig. Bandelari circa l'incarico dato a lui nel r86o d'interessare dei capitalisti d'Europa all'invenzione. Parla poi dell'esplosione e dice di quanto si dovettero indebitare pel loro sostentamento e per le spese della malattia. Continua riferendo che quando il Meucci si rimise alquanto dalla disgrazia toccatagli, ricominciò gli esperimenti, e prese denaro ad imprestito per compilare in inglese (lingua che non gli era troppo famigliare) le carte necessarie e che fece registrare la diffida all'Ufficio dei Brevetti. Riferisce sull'affare Grant, e quindi depone di aver venduto ad un sensale di Clifton, certo John Fleming, alcuni apparecchi del Meucci per trar danaro pel sostentamento, ciò che conferma la deposizione del marito.

La deposizione di Matria Egroff, di Clifton, isola di Staten, dice che egli ha assistito il Meucci in vari lavori relativi alla invenzione di lui ecc., e che verso il 1860 o $186 \mathrm{r}$ il Meucci gli disse che scopo dei suoi esperimenti era di riuscire a trasmettere la voce umana per mezzo dei fili elettrici. Accenna al periodo che seguì l'esplosione e dice che gli apparati visti da lui in casa di Meucci sono dello stesso genere, forma e materia di quelli che vide nel $1859-60$ e $186 \mathrm{r}$.

La deposizione di Samerle Lewis, di Clifton, della Congregazione dei poveri, testifica della povertà di Meucci nel 1880.

La deposizione di W. BowEN, di Clifton, testifica della povertà di Meucci nel 1878 e 1879 , e che la Contea gli passava 25 dollari all'anno come risulta dai registri.

La deposizione di ENrICo KrNG, di Stapleton, testifica delle ferite toccate al Meucci nell'esplosione, delle cure apprestategli come medico, ed accenna alla condizione economicamente e fisicamente misera di lui.

La deposizione di John Flemrng conferma quella della moglie del Meucci circa la vendita degli apparecchi e circa la loro forma, dice che ce ne era da riem- 
pire una botte, e che fece ricerca di alcuno di essi nel suo deposito di robe vecchie senza risultato.

La deposizione di Nestore Corradi, residente a New York di professione artista, dice che verso il 1857 o $185^{8}$ fece uno schizzo di disegno per il Meucci, e che in quell'occasione il Meucci gli disse che esso rappresentava « una delle persone poste ai due estremi di un filo elettrico, dalle quali, egli avrebbe fatto trasmettere ad una lunga distanza la voce umana s, e che il Meucci gli aggiunse di essere lui l'inventore, e che lo chiamava un telefono.

La deposizione di Luigi TARTARINI, residente a New York, che conobbe il Meucci all'Avana e lo trovò di nuovo a New York, dice che nel 1849 gli vide fare degli esperimenti per trasmettere * la voce umana sui fili elettrici * e segue raccontando che vide continuare gli esperimenti a Clifton nel 1857 e 1858 . Accenna all'esplosione, dice pure che gli strumenti visti ultimamente nelle mani del Meucci sono dello stesso genere e di forma eguale a quelli vistigli nell'isola di Staten nel 1852-53-57 e nel 1859, e parla della povertà dell'inventore.

La deposizione di REUBEN LORD di Clifton, testifica della povertà del Meucci.

La deposizione di ENRICo BANDELARI attesta l'esattezza delle informazioni rirca il suo viaggio in Europa, circa l'aver egli veduto nel 1860 ciò che si chiama ora telefono, e che aveva per mira di far parlare fra di loro due persone distanti, e circa l' essere stato richiesto di organizzare una Compagnia, od ottenere il concorso di qualcheduno.

La deposizione di GrUSEPPE GrEgori attesta la ristrettezza dei mezzi dell'inventore.

La deposizione di Antonio Luzzar tende allo stesso scopo.

La testimonianza di PATRICK KeHOc di Clifton afferma che il Meucci gli disse che era dietro a fare uno strumento per trasmettere la voce umana e ciò era nel I861 o I862, ed intese a traverso dei fili del Meucci, il suono della sua voce senza però capire le parole, e che detti fili erano in comunícazione con una batteria galvanica.

La deposizione di JoHN LIDDEL, agente del fu Carlo Chester di New York attesta l'esistenza nei libri dei conti di detto Carlo Chester delle segnenti note:

4 Ad Antonio Meucci, di Clifton Brewery, isola di Staten, Io agosto 1858 , sette paia di magneti su una base sola, dollari 5o. Pagati il 2 Agosto $185^{8}$.

« Ad Antonio Meucci, di Clifton Brewery, isola di Staten, 14 agosto 1858 , dodici elementi di pila Grove a dollari 1.50, dollari I8. Rimonta di magneti e sostituzione dell'anima dollari 42 (totale dollari 60 ). Pagati il 20 Agosto 1858 .

La deposizione di GAETANo NEGRETri dice che nel 1844 si recò all'Avana fece acquisto di alcune batterie galvaniche dal fratello Enrico Negretti Zambra di Londra, e le vendè, insieme ad altri strumenti elettrici, ad Antonio Meucci e più tar di in New York, nel 1851 , fece per suo conto altre provviste in quel genere. 
La deposizione di E. F. SECCHI DE CASILI, residente a New York dice che egli era l'editore del giornale bi-settimanale 1'Eco d'Italia che si pubblicava a New York, e fu informato nel $185^{8}$ dal Meucci che egli era il primo inventore del telefono, e che rese pubblico ciò nel 1859 . La raccolta dei giornali fu poi distrutta.

La deposizione di Ferdinando DE LuCA, già Console Generale nella City di New York dal 1867 al 1878 ed ora (Luglio ${ }_{18} 880$ ) Ministro residente d'Italia in China, asserisce che egli conobbe il Meucci molti anni prima che andasse a New York, e sapeva che egli aveva fatto parecchie scoperte, e che molti anni prima che si recasse a New York, e durante la sua residenza quivi, il Meucci aveva fatto esperimenti per giungere a trasmettere la voce umana per mezzo dei fili elettrici, ciò che si chiama ora telefono. Dà pure ragguagli sulla sua povertà e sui soccorsi prestatigli da lui e da un altro signore.

La deposizione di ANGelo Bertolino (sopra menzionato) contiene una conversazione di lui col Sig. Grant predetto.

La deposizione di L. D.Cunningham di New York, dice che conosceva il Meucci da molti anni e che nel 1877 s'andò a stabilire a New York, e si fece rimettere le carte dal Meucci per farne conoscere il contenuto a uomini di scienza tra cui il Prof. Parmlee, che ciò egli fece perchè ci doveva essere una esposizione alla Steinway Hall verso il Settembre 1877 . Il Prof. Parmlee avendo visto il Caveat fatto inserire nell'ufficio dei brevetti e la descrizione, espresse la sua meraviglia e disse: a se tutto ciò è verità, Meucci ha il diritto di priorità $\nsim$ o parole simili. La deposizione ricorda lo Stetson, colui che fece registrare 1 'invenzione verso il 1876, come compagno di Bell, Gray ecc. 
V.

\title{
IL "CAVEAT ” LO SCHEMA DI A. MEUCCI E LO SCHEMA BELL
}

La documentazione essenziale della scoperta del Meucci, come venne sviluppata e presentata nella sua ulteriore forma di pratica applicazione, è data dal - Caveat " (domanda provvisoria di brevetto) redatto e consegnato dal Meucci stesso all' Ufficio delle patenti di Washington il 28 Dicembre 1871 , come dalla seguente ricevuta: (I)

\author{
DIPARTIMENTO DELI' INTERNO \\ UFFICIO DELLE PATENTI DEGLI S.U.
}

Waskington 28 Dicembre 1871

Signore,

Il vostro * Caveat s sopra un dichiarato perfezionamento sul * Telegrafo sonoro , è stato ricevuto e debitamente registrato negli archivi confidenziali di questo Ufficio.

\section{Rispettosamente vostro}

\section{A. D. LIGGeT}

Ad Antonio Meucci

Commissario

presso Thos. D. Stetson

16, Murrars Street - New York

Riproduciamo qui appresso il documento nella sua integrità coi disegni che vi erano allegati :

La figura prima rappresenta un portavoce concavo, posto sopra un tubo $A$ nel quale tubo è adattata la calotta $B$; attraverso il centro della calotta $B$ passa un elettro magnete tenuto fisso col mezzo della vite $S$. Il diaframma indicato

(x) II \& Caveat n (paragonabile allo \& Specimen provvisorio $n$ ing ese) è definito nella legislazione american a delle patenti come < un avviso trasmesso dall'inventore al Commissario Capo dell'Ufficio Brevetti per impedire entro il termine di uns o due anni il rilascio di un brevetto ad altre persone per la stessa invenzione (Art, 183 del regolamento 17 Giugno r907) - II Caveat venne poi abolito nel 1912. 
dalle linee punteggiate nel punto $D$ è fatto di una sostanza capace d'induzione e la linea $O$ rappresenta 1 'orifizio che gli sta di contro.

Specificazione. - La seguente è una descrizione dell'invenzione che si crede sufficiente per lo scopo di questa diffida:

- Io mi servo del noto potere conduttore dei circuiti metallici continui come canale del suono, e ne aumento l' effetto elettricamente isolando il conduttore e le persone che stanno in comunicazione. Esso forma un telegrafo parlante senza

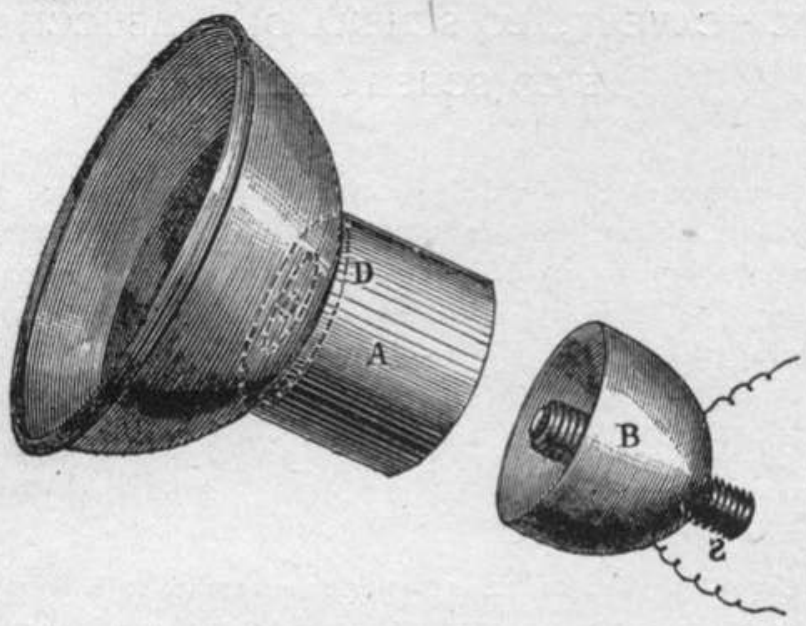

Vista del telefono trasmettitore Meucci.

(Dalla EI. Rew.)

che si abbia a rícorrere ad un tubo vuoto. Tengo a dichiarare che tutto o parte dell' effetto può realizzarsi nello stesso modo che con un tubo metallico.

* Credo che alcuni metalli serviranno meglio di altri, e mi propongo di provare ogni genere di metalli.

- Il sistema che io mi propongo di attuare, ed al quale ho rivolto le mie esperienze, consiste nell' isolare due persone, poste ad una considerevole distanza una dall' altra, situandole su isolatori di vetro, servendomi per esempio: di vetri messi sotto le gambe della sedia o della panca su cui le persone sono sedute, e mettendo queste persone in comunicazione fra di loro per mezzo di un filo telegrafico. Io credo preferibile un filo di sezione maggiore di quella dei fili usati ordinariameute nei telegrafi, ma mi riservo di far esperimenti in tal riguardo. Ognuna di queste due persone tiene alla bocca uno strumento, analogo ad una tromba acustica, entro cui si possa pronunciare comodamente le parole ed il suono si concentri sul filo. Un altro strumento si applica all'orecchio per ricevere la voce dal corrispondente dell' altra parte. 
- Questi strumenti, ossia l'apparato per la bocca e quello per 1'orecchio, sono in comunicazione col filo a breve distanza dalla persona. L' apparato per ascoltare essendo di forma convessa a guisa di una campana di vetro racchiude tutta la parte esterna dell' orechio agevolando e rendendo comoda l'operazione. Lo scopo
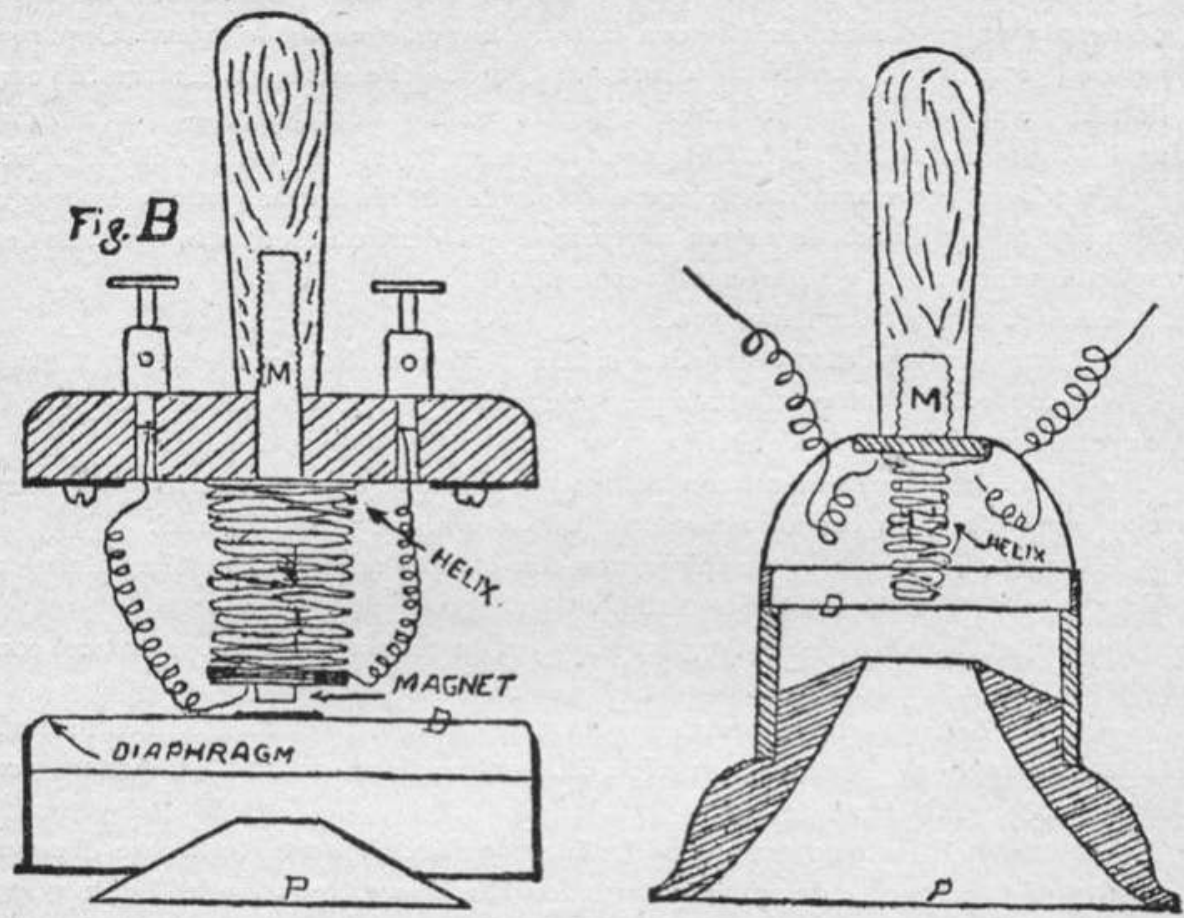
Ricevitore.

Disegni del telefono Meucci (allegati al Caveat).

Trasmettitore.

(Dal Corriere d'America)

è di portare distintamente all'udito le parole della persona posta all' altro lato del telegrafo.

* Per chiamare l'attenzione, il corrispondente può essere avvertito con un segnale od una serie di segnali di un telegrafo elettrico. Ma l'apparato per ottenere questo scopo ed il suo maneggio è molto più semplice che un telegrafo ordinario.

* Mettendosi a operare col mio telegrafo acustico i corrispondenti dovrebbero trovarsi soli nella rispettiva camera, e si deve préndere ogni precauzione per avere l'ambiente perfettamente silenzioso. 
« La bocca chiusa dell'apparecchio, o tromba, per l'udito, e il chiudere le persone sole nella stanza, hanno per iscopo d'impedire la indebita pubblicità delle comunicazioni. Io credo che in questo modo sia facile d'impedire che la comunicazione sia sentita da altri.

«Sarà forse più pratico di operare stando isolata la persona che manda il messaggio ed in comunicazione con la terra la persona che lo riceve. Oppure si possono invertire le condizioni e lavorare con qualche successo. Entrambi i conduttori o apparecchi per la bocca e per l'orecchio dovrebbero - devono, dirò anzi - essere metallici, e fatti in modo da essere buoni conduttori dell'elettricità.

- Reclamo come mia invenzione e domando che sia tenuta come tale per ogni effetto di questa diffida la nuova invenzione qui dentro descritta in ogni suo dettaglio, combinazione e sotto combinazione.

« E, più dettagliatamente, specifico:

* Primo. Un conduttore acustico continuo isolato elettricamente.

« Secondo. Lo stesso adattato per telegrafare per mezzo del suono o per la conversazione tra persone elettricamente isolate già poste a distanza.

" Terzo. L'impiego di un conduttore acustico che è pure un conduttore elettrico come mezzo di comunicazione per suono tra due punti distantí.

*Quarto. Lo stesso in combinazione con quanto occorre per isolare elettricacamente le parti mittente e ricevente.

« Quinto. Il portavoce o l'apparecchio per la parola in combinazione col conduttore elettrico isolato.

- Sesto. Gli apparecchi per l'udito o recipienti pel ricevimento, fatti in modo da essere applicati contro le orechie, in combinazione con un conduttore acustico isolato elettricamente.

« Settimo. Il sistema completo che include il conduttore elettrico e di suono munito di portavoce e di apparato acustico alle due estremità adatti all'uso specificato.

« In testimonianza di che, io ho apposto qui sotto la mia firma in presenza dei due testimoni sottoscritti.

-Antonio Meucci

\section{«Testimoni:}

\section{SHIRLEY M. ANDREW}

FRED « K. HARPER 》.

« I871. Diffida (Caveat) N. 2335 Antonio Meucci, di Clifton, Contea di Richmond, Stato di New York (telegrafo acustico ». Ricevuto il 28 Dicembre 1871 ; domanda del 28 Dicembre 1871 . Dichiarazione del 28 Dicembre 1871 ; descrizione dettagliata del 28 Dicembre 187 r. Tassa, dollari 10. Fatto, H. M. N., E. A. M. * 
Lo schema prospettivo delle connessioni presentato dal Meucci, sebbene in forma primordiale come è riprodotto dalla El. Rew. e da altre pubblicazioni, e la stessa relazione annessa al Caveat, sebbene non esposta nei termini e nei dettagli tecnici, come poterono fare gli altri inventori che erano tecnici e scienziati veri e propri, suggeriscono alcune considerazioni:

Il Meucci conobbe ed applicò evidentemente la reversibilità del suo strumento, ed in realtà l'asta calamitata, il rocchetto e il diaframma vibrante erano identici

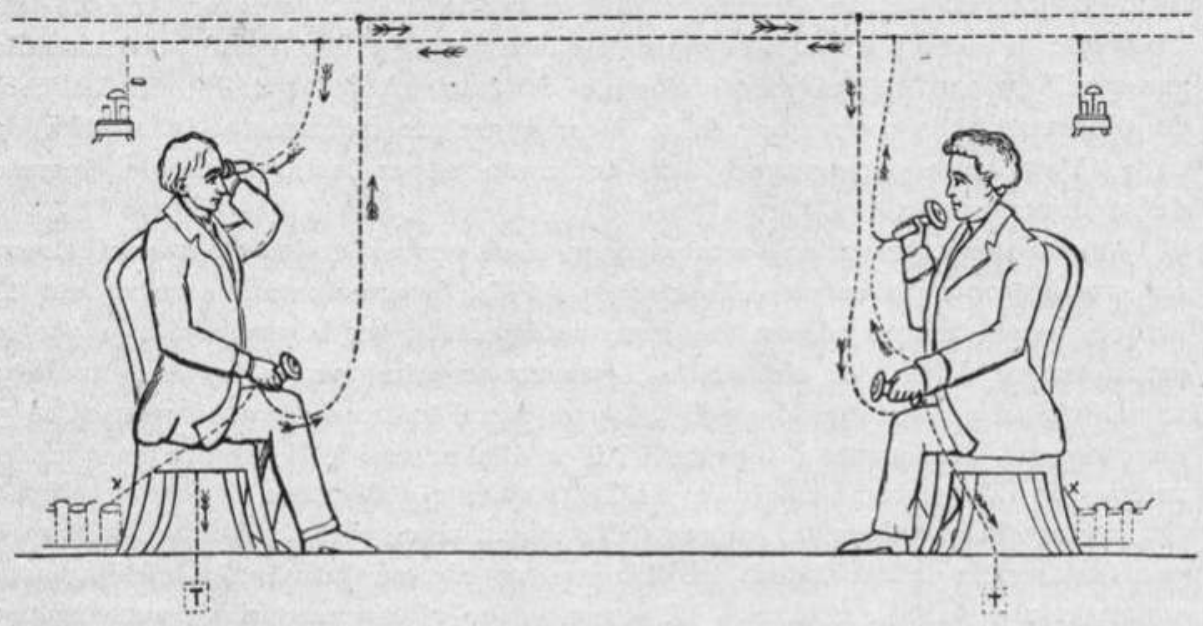

Prospetto e schema della trasmissione telefonica Meucci.

(Dal El. Rew.)

tanto nell'apparecchio trasmettitore, quanto nel ricevitore; ed anche la forma esterna dell' involucro è quasi identica; soltanto che, per agevolare rispettivamente la trasmissione e l'audizione, il primo ha un imbuto e l'altro un padiglione, come nei sistemi moderni, il Meucci inoltre si serve della messa a terra come nel telegrafo per chiudere il circuito di comunicazione.

Non di meno egli adopera nel suo schema e nelle sue esperienze due fili e precisamente uno per ciascun senso di trasmissione, e con questo sistema, e con due semplici apparecchi a portata di mano, egli risolve il problema di poter trasmettere e ricevere contemporaneamente da entrambe le stazioni estreme, ciò che non poteva allora diversamente realizzarsi praticamente ed efficacemente con l'inserzione di due trasmettitori e di due ricevitori, per la tenuità della trasmissione, non disponendosi allora nè del microfono, nè della bobina di induzione.

Questo particolare importantissimo della trasmissione contemporanea in entrambi $i$ sensi non era dapprima prevista dagli altri inventori; e cosi pure gli altri inventori non si preoccuparono del segnale di chiamata, mentre il Meucci prevede 
un apparecchio di soneria o di segnali - più semplici del telegrafo come egli dice - che veniva inserito come in un'impianto telegrafico su uno dei due fili. Lo schema non indica come era realizzata questa inserzione od esclusione dell'apparecchio di chiamata, ma probabilmente ciò doveva avvenire, mediante qualche commutatore od interruttore, analogamente a quanto si fa oggi per e passare a Morse », come suol dirsi, il circuito telefonico per esigenze di servizio.

Tutto quanto potesse occorrere in pratica aveva dunque previsto fin da principio il Meucci nel suo schema, a differenza degli altri inventori, cioè la trasmissione in ambo i sensi, il segnale di chiamata, e perfino, come accenna nella sua specificazione, la maggior sezione e conduttività del metallo più indicato da adoperare nella costruzione della linea, il perfetto isolamento, la sordità da dare all'ambiente per una migliore audizione e per la garanzia del segreto della conversazione.

Ancora una osservazione sembra opportuna per rendersi conto come al Meucct sia sorta la prima ispirazione nella scoperta del Telefono: come abbiamo veduto A. Meucci aveva una tendenza, sebbene rudimentale, per la meccanica e per le esperienze di fisica e di elettricità. Il portavoce primitivo, a filo teso tra due membrane di pergamena, che egli adoperava nel teatro di Avana verso il $18_{35}$, deve avergli certamente prospettata l'idea e la necessità di trovare un qualche mezzo per poter parlare meglio e più praticamente a distanza.

Portata la questione nel campo della trasmissione elettrica, manca purtroppo qualsiasi scritto dell' inventore, nè si hanno notizie, nè dati tecnici, all'infuori di quanto risulta dagli «Affidavit , nè si conoscono gli apparecchi, sia pure primordiali, che esplichino la concezione e lo sviluppo delle prime esperienze e dei tentativi del Meucci.

Dalla stampa americana, che in occasione della morte del Meucci rievocava calorosamente la storia e la vita del grande inventore, e specialmente dagli articoli che abbiamo citato del « New York Times » e del « New York World sel I 9

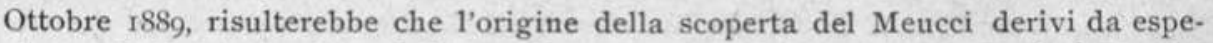
rienze di elettroterapia e della cosidetta «scossa elettrica $»$ con la quale il Me ucci in casa sua curava dei malati : ossia da una piastra in bocca del paziente, egli avrebbre avvertito, attraverso il filo, a qualche distanza la trasmissione di suoni e parole.

Tecnicamente il fenomeno parrebbe a prima vista inesplicabile dato che da una semplice piastra in bocca del paziente non possono derivare correnti indotte che diano luogo a trasmissione di suoni o parole.

Peraltro, sia che si trattasse di una sola lamina o di un solo circuito, cioè con l'intervento della terra, sia che si trattasse di un circuito a 2 fili, una spiegazione potrebbe trovarsi nel fatto che una variazione di resistenza di contatto della piastra, tra la dentatura, la bocca e le labbra dell' individuo abbia potuto effettivamente verificarsi. Il Meucci conosceva ed esperimentava con bobine di 
induzione, già note dal tempo di Faraday ( $\left.183_{32}\right)$ ed era pur noto il rocchetto di Ruhmkorff ( 1851 ) che venne per l'appunto usato nelle applicazioni mediche; ciò forse può spiegare come il Meucci fin da principio con apparecchi e membrane vibranti di fronte ad un elettromagnete, ed inseriti in circuito, abbia potuto come primo indizio rilevare la trasmissione di vibrazioni, rumori e segnali qualsiasi, se non addirittura delle parole vere e proprie.

Schema Bell. Il Dr. Ing. E. Feyerabend Segretario di Stato alle PP. e TT.
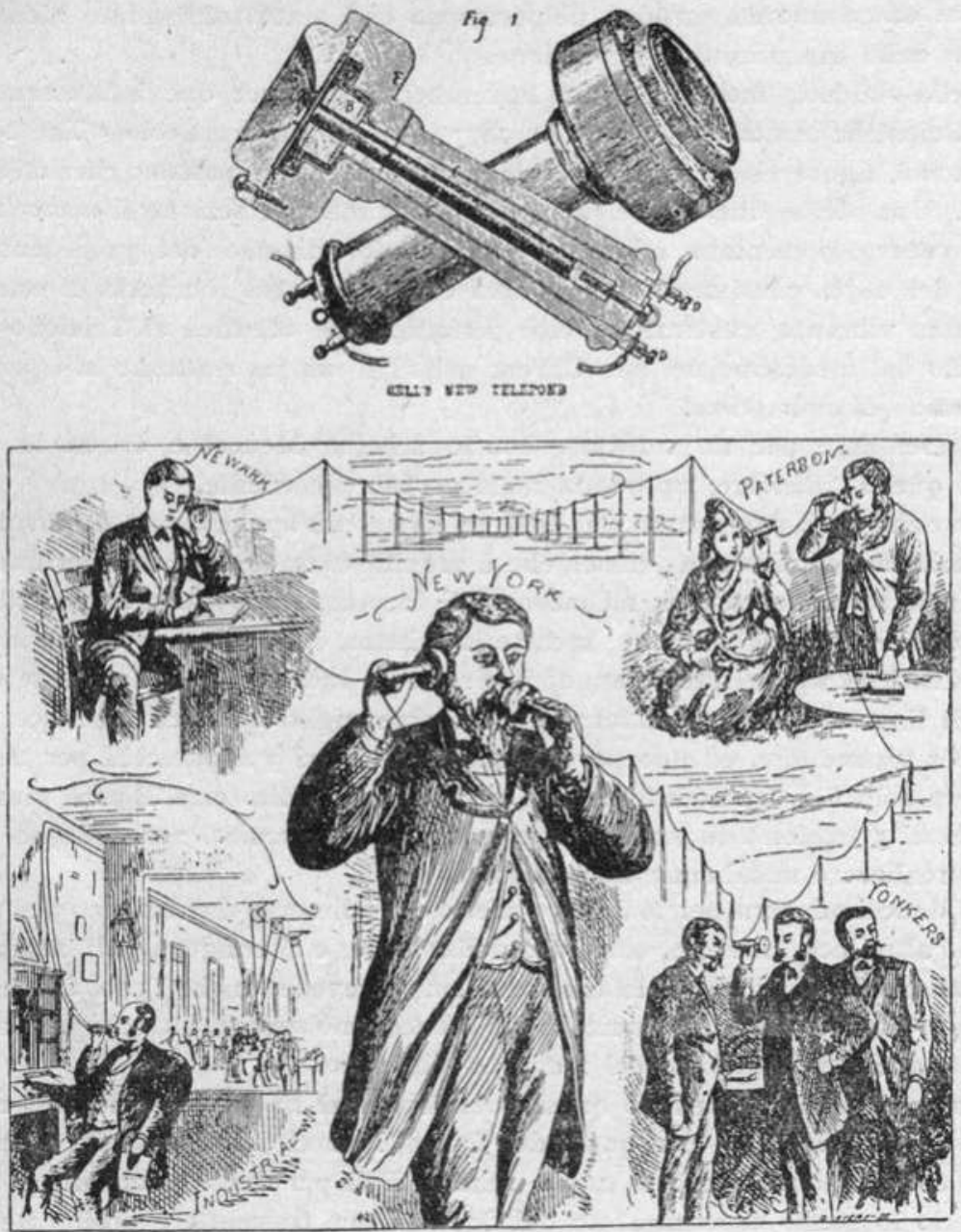

Prospetto e schema della trasmissione Bell (memoria Feyerabend). 
Germaniche in una splendida monografia sulle origini e sulle applicazioni del Telefono in Germania da 50 anni, edita a Berlino nel r 927 presenta con dettagli storici e tecnici l'inizio e lo sviluppo delle prime invenzioni telefoniche (Bell-Gray, ecc.) mettendo particolarmente in evidenza di priorità le scoperte e gli apparecchi di Filippo Reis che è senza dubbio il più profondo investigatore e conoscitore, tra i vari inventori, dei primi apparecchi telefonici. Al Feyerabend però è sfuggito di fare alcun cenno del Meucci, per la evidente ragione che esso è rimasto fino a poco tempo fa quasi del tutto sconosciuto nella stampa e nelle pubblicazioni te cniche americane ed europee, nè purtroppo ci è stato tramandato alcun apparecc hio della sua primitiva invenzione.

Nella suddetta memoria il Dr. Feyerabend riproduce due interessanti figure dello Scientific American del 6 Ott. 1877 inserite in un articolo \& The New Bell Telephon \#, figure che è opportuno richiamare in quanto possono riferirsi a questo studio. Una delle due figure rappresenta per intero e sezione il nuovo Telefono come veniva presentato allora in tipo affatto diverso dai precedenti apparecchi del 1876 , consistente in un'asta calamitata, col rocchetto di estremità e 1a lamina vibrante, costruzione cioè perfettamente identica al Telefono Meucci descritto dal medesimo nel suo Caveat del 1871 , ma gia realizzato ed esperimentato una ventina di anni prima.

Interessante per un raffronto con lo schema Meucci è, altresì la seconda figura, quella inferiore, riprodotta nella pagina precedente.

Lo schema è prospettato dal Bell in forma più appariscente e movimentata: n el mezzo, a New York, la persona che è munita di un apparecchio per trasmettere e di un altro per ascoltare; all'intorno c'è la prospettiva della palificazione con ascoltatori'a Newark-Youkers, mentre lo schema del Meucci si limita a due corrispondenti seduti su due scranne; il circuito è indicato semplicemente con due tratti di linee e la scritta * distanza di parecchie miglia . Il Bell inserisce un telefono per trasmettere, ed uno per ascoltare, con altri 3 apparecchi per ascoltare, sopra un solo filo, in circuito chiuso, senza far uso della terra. É peró presumibile che questi 5 telefoni, disposti in serie in un lungo circuito, non possano praticamente realizzare una buona trasmissione.

Il Meucci adopera per maggiore effetto e rendimento due fili, uno per trasmettere e l'altro per ricevere, con l'uso della terra, e il congegno di segnalazione telegrafica ed acustica per la chiamata, ma prevede anche l'uso di un sol filo senza la terra. La batteria indicata dal Meucci può servire, oltrechè per la segnalazione, anche per rinforzo del magnete telefonico.

La somiglianza, diremmo quasi identicità, del nuovo telefono Bell e del re1ativo schema del 1877 , con quelli del Caveat Meucci del $187 \mathrm{I}$ risulta ad evidenza.

Il collegamento indicato nello schema Bell per due stazioni di un officina, è proprio lo stesso esperimentato dal Meucci per trasmettere ordini nel teatro di Avana e nella fabbrica di birra a Staten-Island. 


\section{PRECURSORI DEL TELEFONO}

Il Maggiore W. C. Barney nella succitata rivista londinese The Telegraphic Journal and Electrical Review, del 23 Febbraio 1884 N. 326 riferisce quanto appresso:

- Le rivendicazioni della priorità d' invenzione del telefono si seguono così da vicino che non è ancora asciutta la penna che scrisse il nome di un primo inventore che già un altro si è fatto avanti per farsi proclamar lui.

- Nella Rivista 7 Gennaio 1882 io pubblicai che Manzetti di Aosta, in Italia, reclamava per la sua invenzione di un telefono nel 1865 , e nel 28 dello stesso mese suddetto, annunciavo eguale reclamo per parte di M. Donough, e si pubblicavano la descrizione ed i disegni del suo brevetto datato dal to Aprile 1876 . Faccio ora pubblico il reclamo di Meucci, un italiano residente a New-York, che asserisce di avere inventato e costruito un telefono nel 1849. Io sentii parlare di questa invenzione, e la trattai come una semplice voce; ma sembra che abbia più fondamento che una semplice voce, perchè Meucci fece registrare la sua invenzione all' Ufficio dei Brevetti degli Stati Uniti nell' anno r871.

- L' estratto seguente di un giornale di Baltimora mostra appunto che si tratta di qualche cosa di più che di voci in questa rivendicazione.

- La Compagnia Globe Telephone di New-York, di cui la Compagnia di qui è il primo ramo che si metterà in attività, è proprietaria del brevetto conosciuto sotto il nome di brevetto Shaw, e sostiene altresi i diritti di Antonio Meucci di NewYork, che si pretende che $\mathrm{sia}$ il primo inventore dell'arte di trasmettere la voce umana attraverso ai fili col mezzo dell' elettricità. E questa pretensione non si basa soltanto sulla pura asserzione di chi crede di avervi diritto; ma i suoi sostenitori sono in possesso delle più numerose e sicure prove della verità dell' asserzione, e possono inoltre dar ragione nel modo più soddisfacente del ritardo nel far valere il diritto alla privativa dell' invenzione. Le prove a cui si allude consistono in dichiarazioni autentiche di molte persone disinteressate che ebbero parte o furono presenti agli esperimenti di Meucci, a cominciare fin dal 1849 andando giù fino al 1875 , e che videro, maneggiarono e provarono il suo apparato s.

- Segue qui una lista di pretendenti all onore di primo inventore del Telefono, in ordine di data delle loro invenzioni. 


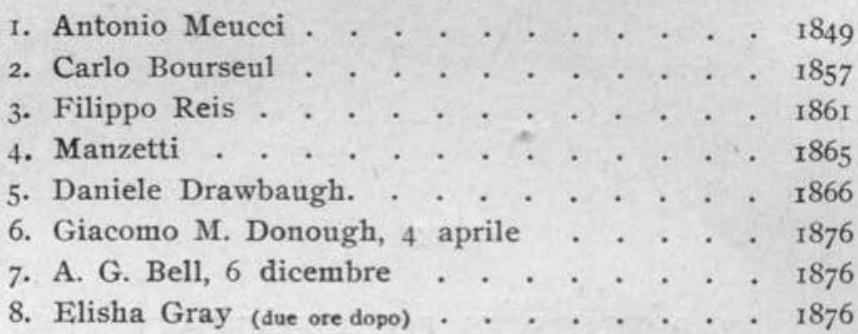

- Il nome di Antonio Meucci sta in capo alla lista, come quello la cui invenzione daterebbe fin dal 1849 come viene certificato da molti testimoni disinteressati. La prima memoria ufficiale di quest' invenzione, per parte di Meucci, è la registrazione da lui chiesta nel 1871 all' ufficio delle Privative degli Stati Uniti, e la prima notizia stampata della sua asserzione "di trasmettere esattamente il discorso" che io abbia potuto trovare, è il Commercio di Genova del r865. Io riassumo dall' articolo di questo giornale quanto segue:

" Essendo stati riprodotti negli Stati Uniti, in ispecie dall' Eco d' Italia del * 19 Agosto 1865, vari articoli che rendevano conto della scoperta fatta dal Man«zetti, della trasmissione del suono e della parola per telegrafo, certo A. Meucci «scrisse al Commercio di Genova una lettera da Clifton, Staten Island, datata da1 «29 agosto r865, nella quale egli reclama la precedenza di fronte al Manzetti. * A quanto afferma egli avrebbe scoperto prima del r860 che col mezzo di un pic« colo strumento collocato nell' orecchio e fatto agire dall' elettricità attraverso ad « un filo metallico, egli poteva trasmettere con precisione la parola tenendo uno « dei capi del filo tra i denti. Con questo metodo due persone poste a distanza « una dall' altra possono tenere una conversazione insieme. Egli aggiunge che con« fidò la sua scoperta nel is6o a un certo Bandelari, suo amico, che stava per par« tire per l'Italia. Nel 1865 , il Signor Meucci, avendo sentito parlare dei risultati «ottenuti da Manzetti, scrisse a Bandelari d'indagare se Manzetti avesse avuto « informazioni di ciò che aveva confidato a lui, Bandelari, prima della sua par« tenza dall'America e il Bandelari gli rispose di non aver comunicato a nessuno la «scoperta che egli si rammentava molto bene di aver sentito esporre in casa del \&Signor Reveccio a New-York. .

- Non mi venne fatto di trovare una descrizione definitiva dei principi su cui si basa l'azione del telefono Meucci; ma ciò sarà in breve conosciuto quando sarà fatto di pubblica ragione il brevetto chiesto da lui nel 1871 negli Stati Uniti ».

Come vedremo in seguito, trattandosi di un caso di specialissima importanza, si presenta una questione di precedenza a favore del $\mathrm{Dr}$. Cushman che nel $185 \mathrm{I}$ ideò e costruì un telefono che avrebbe avuto la priorità indiscutibile sulla in venzione del Bell. Per semplici ragioni di forma, non avendo l'interessato presentato antecedentemente la domanda di brevetto, e non avendo prima fatto co- 
noscere ed applicare la sua invenzione, non potè avere ragione di fronte al Bell; ma la sentenza riconosceva in sostanza la priorità del Cushman.

Non crediamo qui il caso di descrivere dettagliatamente i vari suddetti sistemi : ci limitiamo ad un breve cenno comparativo.

Il telefono Bell in un modello del 1876 era costítuito, come trasmettitore, da una lamina od armatura vibrante mediante una membrana di fronte ad un elet. tromagnete a due poli. In un altro modello la lamina è sottesa ad un imbuto verticale e porta l'appendice di una asta metallica che pesca in un recipiente con acqua acidula. Vibrando la lamina, varia 1 'immersione nel liquido e funziona quindi come variazione di resistenza inserita nel circuito.

In altro tipo si ha la combinazione di due apparecchi di estremità costituiti ciascuno da un elettromagnete, la cui armatura è girevole attorno ad un perno, come gli sportellini indicatori di chiamata dei quadri di commutazione. Tale armatura, o sportellino, posa a guisa di un martelletto sul centro della membrana, che, vibrando per effetto della voce, di fronte all' elettromagnete produce le correnti indotte per interruzioni e variazioni successive di intensità. Viceversa, dall'altro lato, queste ultime fanno vibrare l'ancora e quindi riproducono il suono nella membrana ricevente. L' apparecchio trasmettitore porta un grande imbuto per raccogliere e intensificare la voce di chi parla verso la membrana; 1 'apparecchio ricevitore ha un cono convergente verso l'orecchio per migliorare 1'audizione come nei corni acustici.

Nel sistema Gray il trasmettitore è quasi identico al primo tipo Bell, e cioè ha 1 'imbuto, la membrarra vibrante e l'appendice che pesca in un vasetto contenente acqua acidula, funzionando qui pure come resistenza variabile inserita nel circuito.

Analogamente il ricevitore dell' elettromagnete e la lamina vibrante; questa però è incastrata sul fondo di una coppia, simile ai risonatori Helmoltz, per facilitare 1 'ascoltazione accostandovi l'orecchio.

I due sistemi Bell e Gray si differenziano solo nella forma, ma in sostanza sono identici.

Il telefono Donough è formato da una scatola disposta orizzontalmente, dotata di una membrana davanti alla quale si parla. Alla membrana è annesso un disco con mollette e leve che fanno semplice contatto di pressione sopra una lamina metallica. La membrana, vibrando,produce variazioni nella pressione e quindi nella resistenza di contatto. Il ricevitore consiste in un supporto che regge una tavoletta verticale: su questa è fissata la lamina presentata all'azione di un elettromagnete a ferro di cavallo, e per le correnti indotte riproduce il suono della voce.

Il telefono del Reis era così combinato:

Come trasmettitore era costituito da una cassetta avente da un lato un grosso imbuto per parlare. Sul coperchio era montata la membrana vibrante: questa portava innestata nel centro un punta di platino, prospicente, e vicinissima, ma 
non proprio a contatto, ad una lamina fissa. Vibrando la membrana e con essa la punta di platino, si producevano quindi delle rapidissime aperture e chiusure di circuito, e quindi le oscillazioni di corrente per la trasmissione telefonica.

Il ricevitore consisteva in un sbarra di ferro intorno alla quale si avvolgeva una spirale di filo di rame isolato, percorsa dalle correnti inviate dal trasmettitore.

Queste, con le loro alternazioni ed oscillazioni e smagnetizzazioni deilla sbarra di ferro, producevano, come nelle bobine di resistenza e dei trasformatori, delle vibrazioni elettromagnetiche e conseguentemente dei suoni in corrispondenza con le vibrazioni della lamina del trasmettitore.

L'asse della bobina reso in tal modo vibrante era montato sopra una cassetta di risonanza, ed, essendo in giuoco una intensità di corrente piuttosto notevole, si comprende come il fenomeno si presentasse in forma molto sensibile per la trasmissione di note musicali, suoni, e canto; ma trattandosi principalmente di variazioni di frequenza e di intensità delle vibrazioni, si comprende pure facilmente, come si rilevò di fatto, che il sistema non si prestava alla trasmissione del timbro e delle modulazioni della parola.

Degli altri sistemi Bourseul, Manzetti, Drawbaugh sopracitati si conosce appena il concetto ma è difficile rintracciare i dettagli.

È però indubitato che tutti i varii tipi ora considerati hanno un evidente carattere di apparecchi di fisica e da esperimento, e sono congegni voluminosi complessi e ingombranti e non certo pratici semplici e maneggevoli come quelli del Meucci, la cui forma e composizione, ripresa poi dal Bell, si identifica tuttora negli attuali ricevitori.

Lo stesso telefono Bell si presenta per applicazioni pratiche solo nel suo nuovo tipo del 1877 , precisamente identico al telefono Meucci del 1849 , rivendicato nel Caveat del 187 I.

I primi telefoni costruiti in Europa dalla Siemens Halske, sul tipo Bell, pur unipolari, ma ancora senza microfono e senza accessori, permettevano infatti un'ottima conversazione, in virtù delle dimensioni e della forza dell'asta calamitata, come si espcrimentò qui in Roma stessa, per il servizio di costruzione e manutenzione nello storico impianto di trasmissione di energia elettrica da Tivoli a Roma.

Quegli altri apparecchi inoltre non hanno nei primi tipi la reversibilità, nè gli schemi di collegamento prevedono la trasmissione contemporanea in ambo i sensi, mentre il Meucci fin dall'inizio risolve anche tale questione e realizza anche il segnale di chiamata: prevede ed apre insomma anche la via ad ulteriori perfezionamenti.

È quì opportuno di mettere in evidenza una differenza caratteristica tra la scoperta del Meucci e quella degli altri inventori, del primo tipo Bell é del Gray. Costoro infatti non generavano le correnti indotte, come il Meucci, con una la- 
mina vibrante di fronte ad un magnete, ma si servivano della corrente di una pila o batteria per produrre variazioni di intensità di corrente mediante resistenze a liquido, o interuttori a martelletto, comandati dalle vibrazioni meccaniche di una membrana anche di pergamena. Ed in tal caso i trasmettitori funzionano piuttosto come microfoni e non come generatori di correnti indotte.

Generate tali variazioni di corrente, fu possibile rilevarle con una armatura, lamina o membrana metallica sottoposta all'azione dell'elettromagnete ricevente, come nei primitivi apparecchi telegrafici.

Il Meucci utilizzava la pila, ma solo per maggiore rinforzo ed eccitazione dei magneti di trasmissione e di recezione.

Il Bell trovò il modo di generare la corrente indotta (ondulatoria come si chiamava allora, ma già da altri antecedentemente scoperta) solo nel 1875 , come risulta da una sua lettera al Prof. Ubbard, riportata nella citata memoria del Prof. Kingsbury (Telephone and Telegraphe Exchanges).

In questa lettera scrive il Bell testualmente:

- Incidentalmente ho fatto una scoperta della massima importanza riguardo gli strumenti trasmittenti... Sono riuscito a trasmettere segnali sensa nessuna batteria. La nota musicale prodotta all'estremo ricevente era sensibilmente l'equivalente a quella trasmittente, come altezza di suono o di timbro... *

Il concetto iniziale del Bell, come risulta anche dal brevetto preso in Italia, era dunque principalmente di sostituire ai segnali telegrafici di apertura e chiu. sura di circuito, dei segnali fonici ordinari e quindi di telegrafia multipla. Passò poi il Bell al nuovo tipo di telefono del $1876-77$, trasmittente e ricevente in riversibilitá ed allo schema di trasmissione di cui a pag. 33 , che risultano identici a quelli precisati dal Meucci nel proprio Caveat del 1871 ma già da lui stesso, Meucci, ideato ed esperimentato, in base a documentate testimonianze fin da 11849 .

Ciò costituisce evidentemente un argomento fondamentale di carattere tecnico a favore della priorità nell'invenzione del Telefono di Antonio Meucci.

亡े però doveroso, tra i pionieri soprelencati nella Storia della invenzione del Telefono, aggiungere qualche dettaglio riguardo all'altro italiano sopracitato, Innocenzo Manzetti di Aosta.

Verso il t864 egli realizzò ed esperimentò effettivamente un telefono che destò grandi ammirazioni e meraviglie: ne parlarono vari giornali del tempo, il Diritto di Roma, l'Italia di Firenze, il Commercio di Genova, la Verita di Novara, il Foglio d'Aosta, le Petit Journal di Parigi, e poi il Fanfulla di Roma, e successivamente, come abbiamo veduto, la El. Rev. di Londra nell'882-84, ma non se ne conoscevano i dettagli tecnici.

Nella monografia pubblicata a Torino nel r 897 , l'amico e conterraneo Tibaldi Tancredi narra ampliamente la vita, le vicende e le benemerenze del Manzetti. 
Vi è riportata tra l'altro, rinvenuta tra le carte di un defunto amico del Manzetti, la seguente descrizione della invenzione:

- Le télegraphe parlant se composait d'un cornet en forme d'entonnoir dans lequel se trouvait une lame de fer - en forme de palet très mince - placée transversalement. Cette lame vibrait facilement sous l'impulsion des ondes sonores provenant du fond de l'entonnoir. $\mathrm{Il}$ y avait aussi dans le cornet une aiguille en acier aimanté, enfilée dans une bobine, placée verticalement à la lame vibrante et tout près de la même.

¿ De la bobine, ou fuseau, partait un fil de cuivre enveloppé dans de la soie, dont l'autre bout se reliait à une bobine placée dans un appareil identique à celu décrit ci-devant. De ce second appareil partait un autre fil électriqne qui allait se réjoindre au premier. Or si en proximité de la lame d'un cornet on emettait un son, ce son était aussitôt réproduit par la lame de l'autre cornet .

Si tratta qui dunque, nella sostanza e nella forma, di un vero e proprio apparecchio telefonico del classico tipo della membrana vibrante di fronte ad una elettro calamita, proprio consimile al primissimo tipo del Meucci, ed all'ultimo modello Bell del 1877 .

Una tale invenzione, non desunta da altri, pure essendo stata preceduta ed essendosi incrociata con le analoghe altre prime invenzioni del Telefono, precedente però in ogni caso al Bell, costituisce un grande titolo d'onore pel Manzetti ed un' altra indiscutibile documentazione del Genio inventivo Italiano.

Anche il Manzetti, come Meucci, non ebbe fortuna, nè potè trarre applicazioni industriali e vantaggi economici dalla sua invenzione ed alcune trattative avanzate da ditte americane per la cessione dei diritti della invenzione non ebbero esito.

Il Municipio di Aosta fece scolpire una lapide in onore di lui.

Recentemente, nel Giomale del Dopolavoro PP. e TT. di Roma (Gennaio e Febbraio 1930) veniva rievocata la memoria del Manzetti con interessanti discussioni, mancando però elementi concreti per far risalire al Manzetti medesimo, come riteneva il Dr. Giuseppetti, la precedenza assoluta nelle prime invenzioni del Telefono. 


\section{QVESTIONI DI PRIORITÀ}

\section{NELLA INVENZIONE DEL TELEFONO}

Fin dal 1878 erano stati mano a mano presentati all'Ufficio delle patenti degli Stati Uniti numerosi reclami per la priorità della invenzione del telefono, e precisamente si trovavano in contestazione: Bell - Donough - Edison - Gray - Dolbear - Blake - Irwin - Voelker - Berliner - Richmond - Holcombe - Chinnock - Randal - Phelps.

Il 21 luglio $188_{3}$ l'Ufficio anzidetto notificò le proprie decisioni, con lunghissima e minuziosa relazione di ben 348 pagine. Gli ultimi sei inventori dell'elenco di cui sopra furono messi fuori causa. La complessa e svariata materia che formava oggetto delle contestazioni venne raggruppata in II argomenti e per ciascuno di essi si prendevano in esame $i$ contendenti interessati.

Parecchie delle contestazioni vennero assegnate in priorità a Bell; ma quella del ricevitore telefonico, corrispondente al tipo del telefono Meucci, venne assegnata a Donough (maggio 1875 ) ed era cosi definita "un ricevitore telefonico consistente nella combinazione in un circuito elettrico di un magnete e di un diaframma disposti in immediata vicinanza, con che $i$ suoni lanciati sulla linea possono essere riprodotti perfettamente come intensità e qualità ".

Del brevetto Meucci, ormai scaduto ed obliato, non si parla affatto.

Le suaccennate decisioni dell'Ufficio delle Patenti degli Stati Uniti diedero origine ad una serie infinita di discussioni, polemiche, contestazioni e reclami sia nel campo amministrativo in materia di brevetti, sia nel campo giudiziario con vere e proprie grosse liti davanti ai tribunali. Non è per noi il caso di seguire lo svolgimento intricatissimo di tali vertenze, limitandoci semplicemente a quanto diede poi luogo a riesumare la pratica del Meucci.

A proposito della priorità del ricevitore telefonico riconosciuto al Donough insorsero nel campo tecnico il Du Moncel a favore della priorità di Ch. Bourseul, e Silvanus Thompson a favore del Reis. Il Bell a sua volta sporse reclamo il $\mathrm{I} 7$ Settembre $188_{3}$ contro la decisione dell'Ufficio delle Patenti che riconosceva la priorità del Donough. Ma l'Esaminatore Capo dell'Ufficio Patenti confermava le 
decisioni sopracitate, specialmente quella a favore del Donough, che involge la principale caratteristica del Telefono. Cosi pure il Dolbear e il Drawbaugh reclamarono per la loro priorità. $\mathrm{Si}$ ebbe così un intreccio ed una serie lunghissima di cause e rivendicazioni, che poi finirono col risolversi a favore della Compagnia Bell. Infatti il ig Marzo I888 la Corte Suprema degli Stati Uniti affermava la vali. dità delle patenti Bell: quattro giudici peraltro dissentivano da tale riconoscimento.

In occasione di tali discussioni il già menzionato Dr. W. C. Barney, nella Electrical Review (8 Dicembre $188_{3}$ ) esprime il parere con documentata evidenza che: Bell non fu il primo ad usare la corrente ondulatoria nel telefono parlante (9 Dicembre 1876 ) essendo preceduto da Reis (1861-62) Gray ( 12 Febbraio 1876 ) Donough (1o Aprile I876) e infine Bell non fu l'inventore del Ricevitore telefonico. (9 dicembre 1876 ) essendo preceduto da Bourseul ( 1854$)$, Donough (1875) e Gray (Febbraio 1876 ),

Non disconosce peraltro il Barney i meriti del Bell nell'enorme sviluppo dato al telefono nel campo pratico e commerciale, osservando quanto appresso:

* Per l'attività di Mr. Gardiner G. Hubbard, suocero di Bell, sono stati investiti in compagnie in Europa ed America circa roo milioni di dollari, di cui si sa che il Bell ha ricevuto una parte per oltre 4 milioni di dollari. ?

"Questa certamente è una rimunerazione sufficiente per avere introdotto il telefono al pubblico. Non è certamente giusto che a lui debba essere accordato lonore di essere il primo inventore del telefono privando così i veri inventori dell'onore loro dovuto per le loro magnifiche scoperte: Palmam, qui meruit, ferat .

Il Dumoncel nella Lumière Electrique del 24 Novembre $188_{3}$ sostiene la priorità dell'invenzione Bourseul e conclude che Bell può bensì aver meritato il premio della sua attività, ma non gli spetta lonore della invenzione.

Anteriormente ancora alla invenzione del Bourseul (1857) e del Reis (1864) emerge in ogni caso la scoperta del $\mathrm{Dr}$. S. D. Cushman che accidentalmente, esperimentando uno scaricatore a bobina, scoprì nell'estate del $185 \mathrm{I}$ la trasmissione articolata della parola per mezzo della corrente elettrica, e costrui effettivamente un telefono che, per quanto primordiale, corrispondeva allo scopo.

Instituitasi poi la "Cushman Telephone Company, la Compagnia Bell le mosse causa per infrazione di brevetto. La Corte del Distretto di Illinois a Chicago diede ragione alla Bell, per motivo di forma, perchè il Dr. Cushman non aveva preso a suo tempo il brevetto, non aveva fatto conoscere e non aveva applicato la sua invenzione. In sostanza però la sentenza riconosce e conferma che il merito della scoperta spetta moralmente al Cushman, con una serie di considerazioni che il giudice Blodget svolge nella propria relazione, alcune delle quali meritano di essere quì riprodotte.

* La Corte riconosce che Cushman inventò uno strumento col quale si trasmetteva la parola articolata mediante la elettricitá nel 1851,25 anni cioè prima 
che Bell scoprisse accidentalmente la propria invenzione per un telegrafo multiplo patentato il 7 marzo 1876 , e tradotto poi in vero telefono per la trasmissione articolata della parola con la seconda patente del ro aprile 1876 .

- Ritengo che il Prof. Bell, che indubbiamente è persona onorevole vorrà riconoscere al Dr, Cushman l'onore della scoperta e vorrà chiedere autorizzazione all Accademia Francese delle Scienze di riversare nelle mani del Dr. Cushman il premio dei 300.000 franchi che egli aveva percepito per la propria presunta scoperta.

« I fatti che risultano giudizialmente provati sono:

I). Il Dr. Cushmann costruì un telefono magnetico nel $\mathrm{r}_{5} \mathrm{I}$.

2). Il telefono magnetico descritto e patentato da Bell il 7 marzo 1876 è perfettamente identico a quello del Dr. Cushman nel $185 \mathrm{I}$ e pertanto:

* Al Dr. S. D. Cushman deve essere riconosciuto l'onore di essere il primo inventore del telefono elettrico parlante. Il suo nome deve essere collocato in capo della lista degli inventori del telefono s.

Nelle succitate questioni dı prorità trattate dagli Uffici dei Brevetti e dalle varie Corti degli Stati Uniti, non appare affatto, come abbiamo osservato, la questione del primo effettivo inventore Meucci. Basta qui constatare quanto fosse discutibile la priorità del Bell anche di fronte ad altri precursori. Il brevetto Meucci. ossia il primo Caveat del $187 \mathrm{r}$, a favore del quale, più che mai, per la comprovata precedenza della lamina vibrante di fronte ad un magnete, dovrebbero militare le stesse ragioni messe innanzi per gli altri concorrenti, decadde purtroppo per mancata rinnovazione, e non entrò in discussione, indirettamente, che dopo il 1884 , in occasione della vertenza Globe-Bell, come in seguito si dirà. 


\section{TRATTATIVE DEL MEUCCI CON LA COMPAGNIA DEI TELEGRAFI, CON LA COMPAGNIA GLOBE E CON LA COMPAGNIA BELL}

Abbiamo veduto come le pratiche fatte dal Meucci in Europa a mezzo del suo amico Bandelari, nel 1860, per trovare capitali ed appoggi per lo sviluppo del suo telefono riuscissero infruttuose.

Nè migliore risultato ebbero le trattative del Meucci stesso in America nel I872 con la * New York District Telegraph Company» (come dichiara egli stesso nel proprio Affidavit, e come è confermato nell'Affidavit di Angelo Bertolino) per esperimentare il telefono sui fili di questa compagnia. Il Meucci aveva consegnato la copia del proprio « Caveat » con i disegni e le illustrazioni relative, e dopo 2 anni di pratiche e perditempi, egli non potè neppure ritirare le carte che aveva consegnate, perchè passate non si è potuto sapere a chi ed andate perdute.

La Western Telegraph Union Company (New York Times 25 Settembre 1885) aveva con la Compagnia Bell un contratto con cui si vietava che il servizio telefonico facesse concorrenza al servizio telegrafico della Western Union e si obbligava la Bell a pagare alla Western Union il $20 \%$ cioè dollari r.164.563 come compartecipazione.

$\grave{\mathrm{E}}$ evidente l'interesse di tale Compagnia telegrafica a che la patente Bell non venisse annullata, per non perdere, nella concorrenza, gli introiti della compartecipazione.

La "Chicago Tribune , del 9 novembre 1885 , descrivendo l'invenzione Meucci specifica questi dettagli:

- Dopo che egli (Meucci) depositò il suo * Caveat "ne consegnò una copia ad un uomo faciente parte di una compagnia telegrafica di New York, insieme a disegni e spiegazioni, e quest'uomo promise di fornire i mezzi per fare esperienze dell'invenzione. La storia prosegue rilevando che questo elettricista tenne in sospeso Meucci per due anni, e finalmente, quando Meucci chiese che le carte gli fossero rese, venne informato che erano andate perdute. La tesi della \& Globe Company s (una compagnia che sosteneva i diritti di Meucci) è che i piani vennero mostrati a Bell e questi se ne appropriò le idee.» 


\section{Meucci e la Compagnia Bell}

Già nel $188_{3}$, prima che si iniziassero le varie liti e questioni circa i brevetti e le invenzioni telefoniche, la Compagnia Bell ebbe notizia che stava costituendosi a Filadelfia un sindacato per acquistare i diritti dell' invenzione del Meucci e realizzare l'esercizio del suo telefono. Pensó quindi la Bell ad aprire trattative col Meucci per sventare la concorrenza.

Quattro anni dopo, in occasione delle suddette liti e processi di brevetti il Chicago Evening Journal del 3 I gennaio 1887 rivela al pubblico tali trattative in base a documenti controllati e corredati da regolari « Affidavit ».

Sotto la rubrica \& Dite il prezzo più basso \$ il predetto giornale scriveva:

« La dichiarazione giurata di Mr. Willam W. Goodwin, di Filadelfia, depone che il 2 Agosto 1883 o un giorno intorno a questa data, un gentiluomo chiamato Mr. E. B. Welch, funzionario della Bell Telephon Company, 93 Milk Street Boston Mass, e Presidente della Mexican Bell Telephon Company, scrisse una lettera confidenziale a Antonio Meucci, che era a Staten Island, domandandogli l'esposizione dei particolari della sua invenzione, e chiedendo una sollecita risposta. Mr. Meucci invitò Mr. Welch a rivolgersi ai suoi avvocati e amici Sigg. Lemmi e Bertolino, con lettera in data del 4 Agosto 1883 . A questa, il 9 Agosto Mr. Welch rispose dicendo di desiderare delle copie del Caveat che era stato depositato dall'italiano, e che voleva pure sapere se Mr. Meucci aveva costruito nessun telefono a quell'epoca, prima o dopo la presentazione del Caveat. \& Io vorrei fare un accordo - continua testualmente la lettera del Welch - con voi, » A questo gli avvocati di Meucci risposero includendo copie del Caveat. In conseguenza Welch telegrafò in data $\mathrm{I}_{5} \mathrm{di}$ agosto $188_{3}$ domandando loro \& di dire il prezzo più basso, aggiungendo: è meglio vi teniate pronti ad una offerta: posso vendere quì. » Il I4 agosto Lemmi e Bertolino avevano scritto una lettera che spiegava 1'invenzione di Meucci. Il 25 dello stesso mese gli avvocati telegrafarono al Welch: « ultima offerta ricevuta dollari 180000 ; potete voi aumentare? , Lo stesso giorno Welch telegrafava : - « posso procurarvi una somma maggiore dell'offerta di cui parlate; lettera impostata questa sera.

Tale lettera, pure riportata dal Chicago Evening Journal, non concretava nessuna maggiore offerta, ma dava 1 'impressione di tergiversare. Aggiungeva il Welch:

« Se avete prova buona e sufficiente per stabilire il fatto che Meucci ha fatto un telefono (uno o più) nel I 87 I, 72 e 73 e lo ha descritto nel Caveat depositato all' Ufficio delle Patenti, sono decisamente di opinione che i suoi diritti possono essere 
v enduti per una somma assai più grande di quella nominata nel vostro telegramma, ricevuto questa sera, ossia dollari 180.000 , se il reclamo di Mr. Meucci come è dichiarato nella vostra lettera ricevuta questa mattina, può essere sostanziato con ampie prove. Egli precede non soltanto Bell, ma anche Drawbaugh e Mr. Donough, e dollari 180.000 è una somma troppo piccola per vendere $i$ suoi diritti in questo momento ».

In una riunione fissata al Parker House di Boston avrebbero dovuto conchiudersi le trattative, ma, a causa che gli interessati all'acquisto dei diritti del Meucci erano in quel giorno fuori di città, nulla potè concretarsi, e 1' avv. Lemmi, rappresentante del Meucci dichiara, sotto giuramento, quanto appresso:

* Accettai l'invito, ed esattamente alle ro ant. di quel giorno il cameriere della Parker House mi portò nella camera 49 che mi era stata assegnata, la carta di E. B. Welch. Egli fu introdotto nella mia camera e noi avemmo una conversazione durata circa un'ora, riguardo i diritti di Mr. Meucci come primo inventore del telefono. Io mostrai a Mr. Welch i Caveat e diversi Affidavit; come pure la cronologia del telefono di Meucci. Mr. Welch fece alcune osservazioni su detta cronologia, indicando specialmente la nota e l'Affidavit del notaio A. Bertolino riguardo alla presentazione dei disegni e della descrizione a Mr. Grant, i quali furono perduti, dicendo che egli aveva visto Mr, Grant alcuni anni fa e che Mr. Grant gli aveva detto che quei documenti erano stati restituiti..... ».

Prosegue Lemmi che il Welch aveva chiesto la consegna temporanea dei documenti ma, dato quello che si era già verificato nelle trattative del Sig. Grant con la Compagnia dei Telegrafi, la consegna era stata negata ed il Sig. Welch aveva chiesto allora un rinvio.

$\mathrm{V}$ isto il modo con cui si svolgevano le suddette trattative, queste furono troncate, ed il Meucci ritenne di rivolgersi alla \& Globe Tel. ».

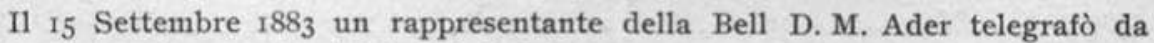
N. Y. ai nuovi proprietari dei diritti del Meucci, Mr. A. P. Willoughby, chiedendo un colloquio ed un appuntamento fu fissato attraverso gli attorneys John $\mathrm{H}$. Caill e C. all'Hotel Doover a Filadelfia: la Bell delegava a rappresentarla anche 1'Avv. George L. Roberts. Ma la Globe, sentendosi in grado di resistere, rifiutò e le trattative furono troncate.

I negoziati di cui sopra, come giustamente rileva il Corriere d'America, dimostrano evidentemente l'importanza che un funzionario della Compagnia Bell e Presidente della Mexican Bell Telephon Company, Mr. Welch, dava alla invenzione del Meucci e l'implicito riconoscimento della precedenza. 


\section{CAUSE E COMPETIZIONI \\ PER LE INVENZIONI TELEFONICHE}

Fallite, come abbiamo veduto, le trattative del $188_{3}$ della Bell con la Globe e il Meucci, la Bell portò la questione nel campo giudiziario, citando la Globe, varî cointeressati in questa compagnia, e personalmente lo stesso Meucci, per violazione del proprio brevetto 7 Marzo 1876 , davanti al Circolo della Corte degli Stati Uniti del Dipartimento del Sud di New-York. La Corte redasse una lunga relazione il 2t luglio 1887 , che concludeva col dar ragione a Bell, perchè il Meucci non avrebbe raggiunto un risultato pratico, 1'invenzione sarebbe stata solo al1' inizio, non era in condizioni di brevetto, e non aveva prodotto nulla di nuovo nell' arte di trasmettere la parola, seguivano altre considerazioni, derivanti probabilmente dalla infelice ed incompleta specificazione del Caveat, mentre i disegni allegati al Caveat precisavano i dettagli tecnici ed il funzionamento dell'invenzione del Meucci. Venne infatti notificata alla Globe ed al Meucci, in data 2 Agosto 1887 l'ingiunzione di non vendere nè usare i "perfezionamenti nella telegrafia * in infrazione del suceitato brevetto .

La cosa destò sorpresa specialmente negli ambienti tecnici perchè effettivamente, nonostante le spiegazioni formali del Caveat, in realtà il Meucci aveva presentato uno schema tecnicamente esauriente. Ciò infatti venne riconosciuto dalla rivista londinese The Thelegraph and Thelephone Journal quando nel fascicolo di Ottobre 1924 , in occasione di polemiche suscitate dalla lapide di Firenze in onore di Meucci, sotto la rubrica «chi fu il primo inventore del Telefono?» riportando la suddetta sentenza, la commentava nel senso che, ciononostante, effettivamente la prima scoperta dell'invenzione del Telefono era in priorità dovuta al Meucci.

La sentenza di cui sopra si prestava quindi certamente ad essere validamente impugnata avanti alla Corte Suprema ; ma, sia perchè erano in corso, e in attesa di decisione, i numerosi altri reclami per precedenza contro le Patenti Bell (Gray, Donough Dolbear e vari altri precursori) sia perchè era aperta l'altra causa giudiziaria per l'annullamento della patente Bell, non vennero mosse ulteriori contestazioni, da parte della Globe contro la succitata sentenza. 


\section{Causa per l'annullamento del brevetto Bell}

In seguito a denunzie di privati, a citazioni da parte della Globe e della Pan Electric Co, a proteste del pubblico e della stampa, ed all'intervento stesso del Governo, sorse pertanto nel 1885 la nuova fase della vertenza per infirmare la validità e legalità della patente Bell e provocarne 1'annullamento.

Nell' agosto 1885 Bell venne denunziato per frode con a Affidavit * dei cittadini W. Van Benthuysen di New-York e Charles P. Kuntington di Mississipi, controfirmato da due avvocati J. R. Beckiwth e Geroge Gautt, diretto al District Attorney W. Nc. Corey, del Tennessee. Da tale lungo documento si stralcia quanto appresso :

* Noi siamo in possesso di informazioni che reputiamo autorevoli, le quali nella nostra opinione, sono sufficienti a stabilire $i$ seguenti fatti:

« Io Che Alessandro Graham Bell, considerato inventore patentato di un affermato utile perfezionamento nella telegrafia e di un affermato perfeziona mento nel telefono elettrico, non fu loriginale e primo inventore dell' arte della tele fonia, o di qualsiasi altra materia sostanziale descritta e reclamata nelle sue lettere di patente.

* $2^{\circ}$ Che il telefono parlante elettrico era conosciuto e usato negli Stati Uniti e in Europa molto prima della pretesa invenzione e scoperta del suddetto.

- $3^{\circ}$ Che egli era a conoscenza di detti fatti, aveva assistito ad esperimenti del telefono parlante elettrico ed aveva nozione della scoperta della prima invenzione.... Che egli non è il fondatore di detta arte e non ha diritto di starne alla testa a ragione della priorità della scoperta.

* $4^{\circ}$ Che il suo giuramento per la domanda di lettere di patente, col quale si proclamava primo e originale inventore di quell' arte era ed è falso ».

L' «Affidavit » procede specificando le accuse, spiega che le pretese scoperte di Bell non erano al più che delle modificazioni del telefono esistente «modificazioni senza valore in se stesse e non usate neppure dai possessori delle patenti del detto Bell, ed al contrario scartate». Quindi precisa che l' Ufficio delle Patenti, arbitrariamente avrebbe dato a Bell la priorità su Gray, mentre i * Caveat, dei due vennero presentati nello stesso giorno.

L'Attorney General, Garland, di fronte alle accuse dettagliate e specificate, ordinò un'inchiesta, il solicitor General Goode fece un rapporto. Accuse gravi furono sollevate contro 1'Ufficio delle Patenti, che avrebbe favorito Bell commettendo gravi irregolarità. Il fatto che Bell riuscisse ad ottenere le patenti in soli 20 giorni parve assai strano. In seguito all'inchiesta, date le gravi risultanze, 1'Attorney General ordinò procedimento giudiziario contro Bell in nome del Governo degli Stati Uniti.

Il corrispondente del Brooklyn Daily Eagle scriveva da Washington : 
"Io non ho visto il documento dell' Ufficio delle Patenti che il Solicitor General ha profondamente esaminato, dandone relazione al Presidente, prima di ordinare l'azione contro la Compagnia Bell, ma formandomi un'opinione da altri fatti conosciuti intorno al Bell stesso, direi sommariamente che le pretese di questa Compagnia sono fondate sopra una frode: cioè sulla pretesa che Bell sia autore del telefono *.

Il Governo degli Stati Uniti, esaminate le accuse e trovatele sufficientemente documentate promosse giudizio alla Compagnia Bell nel Settembre del 1885 .

Riguardo alla gravità di queste accuse il New York Times del 20 settembre $188_{5}$ in un articolo editoriale forniva i seguenti chiarimenti :

- Eे dimostrato che la domanda di Bell era per una patente che coprisse certi nuovi ed utili miglioramenti della telegrafia. Si afferma che i disegni, le descrizioni e le richieste di patente erano così furbamente e artificiosamente scritti e concepiti da fare apparire che si trattasse di miglioramenti della telegrafia multipla armonica, e non di altra materia o invenzione di qualsiasi genere, e che $i$ dispositivi e gli apparecchi che il Bell proclamava adeguati a compiere il lavoro specificato erano inadeguati e senza valore.....

* Appena ottenuta la patente, si afferma, Bell immediatamente pretese e proclamò apertamente che la patente si riferiva non soltanto a certi impraticabili dispositivi da usarsi nel telegrafo multiplo armonico, ma gli conferiva un pieno e completo monopolio della trasmissione delle parole parlate per mezzo dell'azione dell'elettricità.

« Gli Stati Uniti, come parte querelante, asserirono che Bell sotto il mantello e la pretesa di ottenere una cosa, ingannevolmente, e colpevolmente, a cagione in parte della incompetenza e della ignoranza dell' esaminatore, fu abilitato a reclamare un'altra cosa; che il Governo non credeva o supponeva che la patente emessa fosse data per alcuna nuova arte di trasmettere la parola articolata per mezzo dell'elettricità, o che la patente potesse essere a ciò intesa, ma supponeva fosse concessa per miglioramenti nella telegrafia multipla armonica. II Governo, inoltre, asserisce che non supponeva che la patente potesse essere usata per assicurare a Bell il monopolio, che egli ha finora goduto di un' arte conosciuta molto prima che egli depositasse la sua domanda di patenti. In breve, esso dichiara di essere stato ingannato e frodato, con danno del popolo, che è stato privato dei suoi diritti».

Il New York World del 2 Ottobre 1885 , al sorgere delle polemiche contro l'Attorney General Garland riporta la seguente informazione:

..... Cinque anni prima che Gray e Bell facessero la domanda per le loro patenti Antonio Meucci, di Staten Island, depositò un «Caveat» per un telefono parlante, nel quale egli descrisse il telefono ora in uso, ma, strano a dirsi, i suoi disegni sono scomparsi dagli archivi dell' Ufficio delle Patenti. Oltre a cinquanta 
persone, tuttavia hanno rilasciato Affidavit, dichiarando che il telefono Meucci era in uso ed era un buon telefono parlante prima del $1871 \ldots \ldots$.

La Evening Post del ro Novembre 1885 , in occasione delle udienze che si tenevano a Washington davanti al Sottosegretario di Stato Lamar per la causa che avrebbe dovuto intentarsi contro la Compagnia Bell riporta il seguente telegramma :

- La Pan Electric Company ha sottomesso degli Affidavit, di varie personalità scientifiche, che mostrano come il telefono non sia stato inventato da Bell. Gli Affidavit sono dei professori Green, Pickering, Vanderweld, Thomas A. Edison « ed altri s.

Sempre in occasione delle suddette vertenze, nel Novembre 1885 , il giornale The Sentinel di Chicago scriveva in un editoriale: "Antonto Meucci, l' amico e il compagno del Gen. Garibaldi, quando questi era un esiliato nel nostro paese, è senza questione o dubbio il primo inventore del sistema telefonico ora cosi noto ed in uso. .

È opportuno di rilevare che in Europa, a richiesta della Compagnia Telefonica Austriaca, il Ministero Austriaco del Commercio, ed il Ministero Ungarico di Agricoltura Ind. e Com. decretavano poi per loro conto il 28 ottobre 1887 l'annullamento delle patenti Bell.

\section{BIII degli S. U. per promuovere l'annullamento della patente Bell}

Il I5 Marzo . 886 il « Solicitor general , Good degli S. U. promoveva causa contro la Compagnia Bell per l'annullamento delle Patenti 7 Marzo 1886 e 30 Gennaio 1887 in base alla Sez. 8 Art. I della Costituzione.

$\grave{\mathrm{E}}$ interessante rilevare riassuntivamente $\mathrm{i}$ seguenti dettagli del $\propto \mathrm{Bill}$ » (atto ufficiale di accusa) che concludeva chiedendo $l$ annullamento della patente Bell perchè ottenuta illegalmente ed impropriamente (illegaly and improperly procured).

\& L'interessato era in obbligo di dare tutte le spiegazioni, dettagliare l'invenzione ed esporre i precedenti e tutte le notizie che debbono essere manifestate all' Ufficio delle Patenti. La Patente Bell era chiesta in termini ambigui e generali in guisa da celare l'invenzione in questione e le successive e trarre in in. ganno e disorientare gli esaminatori dell' Ufficio delle Patenti: una richiesta in modo cosi delusivo ed ambiguo era ed è vuota e di nessun effetto.

« Le patenti Bell suddette furono ottenute illegalmente ed impropriamente.

« Bell avrebbe conosciuto il testo della patente Gray a mezzo degli ufficiali delle patenti.

« Gray in tali discussioni e contrasti sarebbe stato indotto ad abbandonare il suo Caveat. 
« La corrente ondulatoria non è scoperta da Bell ma da Faraday ed era applicata nel telegrafo Gauss, Weber e Siemens. Bell sarebbe stato preceduto nella scoperta del «Telefono elettrico parlante * da Reis, Varley, Antonio Meucci, Gray Edison, Eaton e molti altri.

* Essendo stata abbandonata la scoperta del primo inventore originale, la scoperta passa in dominio pubblico

- Quando Bell prese la patente del 30 Gennaio 1887 essa non era di sua invenzione e si pretende che sia stata presa per frode ed a danno di Dolbear, perchè questi l'avrebbe rivelata ad un amico di Bell. *

Le succitate denunzie erano spinte dalla pubblica opinione e dalla stampa americana, per ragioni economiche, oltrechè politiche, per il fatto che si calcolava che il monopolio Bell apportasse ai cittadini un aggravio di circa ro.ooo dollari al giorno.

Per avere un'idea della immensa mole delle liti varie che si aggiravano intorno alla Bell, basti tener presente che al 7 Febbraio 1885 tali liti avevano già assorbito una spesa di 200.000 dollari.

Nell' Ottobre 1885 vennero presentate, nel campo industriale le altre 3 domande per 1'annullamento dei brevetti Bell da provocarsi mediante la forma di giudizio americano «equity». Due di queste petizioni erano presentate dalla Globe Telephone Company che si basava sul brevetto Meucci e l'altra dalla Washington Telephone Co. Queste domande furono trasmesse al Ministero dell' Interno per "riferimento e parere" e per raccomandazione del Segretario dell' Interno e questi a sua volta le passò al Commissario delle patenti per " riferimento e parere s. Il Commissario delle Patenti riferì sommariamente al Segretario dell' Interno Lamar il quale ordinò che si sentissero gli interessati con pubblico procedimento e si esaminassero i documenti riferendo sullo stato di fatto della questione.

La Bell, come abbiamo osservato, aveva avuto ragione contro gli altri concorrenti con sentenza degli S. U. a Filadelfia (I9 Marzo I888) che decideva per la validità delle patenti Bell.

$\mathrm{Ma}$, riguardo a queste altre questioni di annullamento, la Corte del Massachussetts decideva che il Governo degli S. U. non aveva diritto di procedere all'annullamento della Patente.

L'Attorney generale degli Stati Uniti mosse appello contro la decisione del Massachussetts, appello validamente sostenuto dall' Avvocato Capo A. G. Thurman, candidato democratico alla vice presidenza degli Stati Uniti.

" La Suprema Corte decise in favore del Governo nella causa degli Stati Uniti contro la Bell C. portata in appello contro la decisione della Corte Federale del Massachussetts, la quale sosteneva la eccezione sollevata verso la causa intentata dal Governo per cancellare la patente con la motivazione che essa era stata ottenuta per frode e che il Bell non era il primo inventore. 
« La Suprema Corte rigetta il gindizio della Corte del Circolo del Massachussets e rimanda il caso per giudizio ad altro rribunale.,

Non è stato poi possibile trovare traccia del seguito e della sentenza definitiva, non ritrovandosi più le carte relative agli Uffici delle Patenti degli S. U., che probabilmente per il lungo tempo trascorso saranno andate disperse e non se ne è potuto trovare copia in quegli archivi.

In ogni modo la succitata sentenza riconosce già di per se stessa un serio fondamento per la ulteriore discussione di annullamento della patente e della conseguente priorità del Bell.

Ormai la Compagnia Bell aveva esteso enormemente i propri impianti : dopo appena due anni, nel 1891 , la patente scadeva naturalmente e la questione della priorità del brevetto sarebbe passata in seconda linea. Fin dal 1887 in un'Assemblea degli Azionisti, la Compagnia Bell dichiarava: « L'immenso affare è stato attuato e la Compagnia ha acquistato tale forza finanziaria da poter considerare tranquillamente, qualunque abbia ad essere, la decisione della Corte Suprema, anche se si trattasse di una limitazione o dell'annullamento della patente Bell *

Come abbiamo veduto, la voce che fosse intervenuto un accomodamento contro un compenso al Meucci di L. I00.000 dollari, è smentita da fatti e dallo stesso Meucci che purtroppo nell' Ottobre del 1889 moriva povero, attendendo ancora come scriveva al fratello nelle sue ultime lettere un riconoscimento ufficiale ed un compenso economico del suo diritto di inventore.

(Vedere in appendice la lettera di Giuseppe Meucci). 
$\mathrm{x}$.

\section{LA PATENTE BELL IN ITALIA}

Il Brevetto Bell venne registrato in Italia il 27 Novembre 1877 Reg. Gen. Vol, ${ }_{13}$ No 9647 come: Perfectionnements dans la telephonie electrique inventés par $A$. G. Bell des Etats Units d'Amerique.

Questo brevetto, riguarda, principalmente, trasmissioni di segnali di telegrafia multipla sopra uno stesso filo, ed in ambo i sensi, mediante diverse note musicali. L'antecedente patente americana del Bell del 7 Marzo 1876 No ${ }_{174465}$ era basata sopra \& una membrana tesa alla quale è applicata l'armatura di una elettrocalainita, per mezzo del quale strumento il movimento può essere impresso all'armatura dalla voce o da strumenti musicali * con una successione di impulsi elettrici differenti per velocità, alimentati da una batteria.

In questo « brevetto del 1877 , alla membrana è sostituita una placca di ferro o di acciaio, suscettibile di essere messa in vibrazione dai suoni prodotti in sua yicinanza e quindi dar luogo a correnti indotte, senza batteria.

Per quanto riguarda la parte telefonica propriamente detta, che nel brevetto occupa l'ultimo posto, è riportata questa specificazione:

* Cinquièmement: La formation, dans un téléphone électrique tel que cela est représenté et décrit, d'un aimant avec une bobine sur la ou les extremités de l'aimant le plus près de la plaque .

Dopo altri dettagli di forma, di regolarizzazione, ecc. prosegue:

* Multierement: La combinaison, dans un système de téléphone electrique, d'un aimant permanent avec une plaque en fer ou acier ou autre matière susceptible d'action inductive avec des bobines sur la ou les extremités du dit aimant le plus rapprochées de la plaque, essentiellement, comme il a été décrit. »

Si parla anche qui, posteriormente, di riversibilità tra apparecchio trasmettente e ricevente, ma l'apparecchio come è rappresentanto dal disegno, è ben lungi ancora, pur nel 1877 , dalla forma pratica ideata dal Meucci, mentre è ad esso identico in sostanza nell'idea e nel modo di agire sopra specificato. Qui inoltre si parla di possibilità e di esperienze, mentre il Meucci aveva già ottenuto risultati concreti messi in evidenza, nello schema e nei dettagli, nel suo Caveat del $187 \mathrm{I}$, del proprio e vero telefono. 
L'apparecchio Bell, come risulta dal disegno allegato alla Patente, ha la forma di una cassetta, quasi identica al tipo Reis, posata su una scatola di risonanza, contenente la calamita e la placca vibrante incastellata sopra complicati supporti e viti di regolazione. Dalla cassetta sporge un grosso tubo con un imbuto all'estremità per la trasmissione.

Ha insomma tutta l'apparenza e la sostanza di un apparecchio di fisica, di un telefono affatto primordiale, diverso dal semplice, pratico e maneggevole telefono che troviamo disegnato nello schema Meucci del ${ }_{1} 8_{71}$, già da lui costruito ed esperimentato tanti e tanti anni prima.

Col suaccennato brevetto del 1877 , la « International Bell * di New York con la Corrispondente « Telephone Bell » di Anversa, conquistò di fatto il Monopolio telefonico in tutta Italia ed ottenne verso il I88o concessioni di impianti ed esercizio di reti telefoniche nelle principali città, Milano, Torino, Genova, Venezia, Bologna, Firenze, Roma, Napoli ed altri centri minori.

Le concessioni telefoniche erano accordate, anche in una medesima città, in regime di concorrenza privata, ed erano sorte diverse Società, la Ligure, la Lombarda, la Piemontese, la Telefonica per 1'Italia Centrale, la Generale Italiana, la Romana e la Meridionale, che con diversi tipi di telefoni operavano in concorrenza tra loro stesse e con la Bell medesima nelle sue ramificazioni locali in Italia, specialmente a Torino, Genova e Milano.

La Bell però, minacciando di intentar lite per presunto abuso ed infrazione di brevetto, indusse le altre Società a venire con essa ad accordi. Essa infatti cedette in ripartizione ad esse l'uso del proprio brevetto e del brevetto Blacke, e cedette altresí le reti costruite e le concessioni ottenute in Italia, rimanendo però fortemente interessata con le Sòcietà telefoniçe di allora, e con quelle che ne derivarono, fino agli ultimi tempi, per apporto di azioni, preferenza di esecuzione di lavori e forniture di materiali nelle reti cedute, e nell'ulteriore grande sviluppo delle concessioni e degli esercizi telefonici. 
XI.

\section{CONCLUSIONE}

Dai documenti, dalle testimonianze e dalle varie notizie sopra minutamente riportate, e cioè dal * Caveat s del Meucci e dagli annessi * Affidavit * dalle notizie fornite dal New York Times, dal World, dalla Chicago Tribune, dalle dichiarazioni Ulman, dagli elementi esposti dalla Elettrical Review, e dal Corriere d'America, risulta indiscutibile che Antonio Meucci fu veramente, come egli stesso rivendicò, il primo, tra i pionieri dell' invenzioni telefoniche, che nelle proprie concezioni ed esperienze, nel ${ }_{1} 84$ intuì e realizzò la trasformazione e il trasporto della voce umana mediante le correnti elettriche.

E precisamente è dovuto a lui quel telefono reversibile, ancora in uso nella moderna telefonia, il quale in forma semplice, pratica e maneggevole, è costituito, nella sua composizione caratteristica, dalla classica membrana di fronte ad un magnete, come venne descritto nel «Caveat, del 28 dicembre 1871 e come è stato adottato nelle ulteriori applicazioni.

Il Meucci, come dapprima ideò, lavorò poi intensamente intorno al suo apparecchio con la passione della invenzione scientifica, ma non afferrò subito l'importanza dello sviluppo industriale che ne poteva derivare. Non ebbe fortuna nei primi tentativi di trovare fondi e capitali per finanziare e sfruttare la sua invenzione; restò postergato, deluso, forse anche artificiosamente, nelle sue trattative con la « District Telephone Company * di New York e con la stessa Compagnia Bell.

Allorchè poi si appoggiò alla «Globe Telephone C. * si trovò impelagato nella serie di liti, che di fronte ad una compagnia potentissima ed enormemente sviluppata, quale era la Compagnia Bell, non condussero ad alcun risultato pratico, all'infuori di far rievocare dal lato morale il merito della prima invenzione del nostro grande e pur sfortunato ed incompreso Meucci.

$\mathrm{E}$ che proprio il Bell fosse stato il primo inventore, è fortemente contestato nella sostanza dei documenti sopracitati, e dalle stesse contestazioni legali degli altri precursori - pur posteriori al Mencci - nonchè dalla motivazione delle sentenze, dal parere di autorevoli personalità, e dalle stesșe cause promosse per l'annullamento del brevetto Bell. 
Mentre fervevano nella stampa inglese ed americana le famose discussioni e polemiche prevalentemente a favore del Meucci, si domandava qualche giornale come mai la grande scoperta di lui non avesse raggiunto l'adeguato successo e fosse rimasta perfino in oblio per tanti anni; fino al 1884 , al tempo cioè dell'Esposizione di Filadelfia allorchè ebbero inizio le note cause e contestazioni di inventori e di compagnie di telefoni.

La Chicago Tribune del 9 novembre 1885 spiega la cosa altrettanto semplicemente che esaurientemente, con quanto appresso:

- L'obbiezione generalmente sollevata contro la credibilità della storia di Meucci è che l'inventore non avrebbe permesso che una materia cosi importante rimanesse tanto a lungo nella oscurità, ma gli amici del suo reclamo richiamano l'attenzione sul fatto che egli era estremamente povero - cosi povero che era costretto alle volte di accettare l'aiuto pubblico - , che egli non poteva parlare inglese, e che proprio dopo di aver depositato il suo Caveat egli fu seriamente ferito dall'esplosione del ferry-boat Westfield. In quell'epoca, inoltre, era quasi impossibile interessare dei capitalisti in qualsiasi cosa di quel genere, così lontane erano simili cose dell'esperienza della gente di quei giorni. Frederick Gower, che intraprese la ricerca di capitali per Bell, quando il suo strumento giunse alla conoscenza del pubblico, dichiarò in questa città, circa due anni fa, che egli lavorò quattro o cinque mesi cercando di ottenere un appoggio finanziario, prima di avere qualsiasi successo. E questo avveniva in periodi in cui l'utilità della macchina era stata dimostrata alla presenza di larghe udienze in sale pubbliche. Così non è affatto sorprendente che Meucci fosse così lento a portare fuori la sua invenzione. ,

L'Electrical World mettendo in evidenza analoghe considerazioni e circostanze di fatto, definisce come un pietoso romanzo la storia del Meucci e conclude: " La sua storia è un altro esempio della contrarietà della sorte provata dai pionieri in ogni ramo e della jattura che si continuerà a constatare finchè vi saramno degli inventori su questa terra. ,

Tanto più meritori e sorprendenti emergono nella storia dell'invenzione del Telefono le primissime esperienze e $\mathrm{i}$ primissimi tentativi e risultati raggiunti dal nostro Meucci, in quanto che egli non poteva disporre nè della coltura, nè delle cognizioni tecniche e scientifiche, nè dei mezzi di laboratorio, nè degli appoggi finanziari e materiali di cui potevano poi avvalersi, pure indiscutibilmente degni del più grande onore e della universale gratitudine, i di lui successivi competitori. 
APPENDICE I.

\section{BIBLIOGRAFIA E DOCUMENTAZIONE}

\section{Riviste tecniche}

$1 l$ Telegrafista - $1884-1889$ - Roma

Rivista Telegrafi e Telefoni - 1922 (fasc. 4) 1923 (fasc. 5) 1924 (fasc. 6) - Roma La Lumière Electrique - 24 novembre ${ }_{18} 88_{3}$ - Paris

L'electricien - $188_{3}$ - Paris

The Telegraphic Journal and Electrical Review - Londra 1882 - 28 gennaio

1883 - 21 luglio - II agosto - 20 ottobre - 8 dicembre

1884 - 23 febbraio - 1 marzo - 11 ottobre - 22 novembre

I885 - 18 luglio -25 luglio $\quad-1$ agosto -8 agosto - 15 agosto

22 agosto - 5 settembre - 26 settembre - 3 ottobre - 10 ottobre

17 ottobre -31 ottobre -14 novembre -28 novembre - 12 dicembre

19 dicembre - 26 dicembre

1886 - I gennaio - 15 gennaio - 22 gennaio - 29 gennaio - 12 febbraio

19 febbraio - 26 febbraio - 5 marzo - I2 marzo - 26 marzo

9 aprile $\quad-23$ aprile $\quad 4$ giugno - II giugno

1888 - 6 gennaio - 2 marzo - 23 marzo - 26 aprile - 17 agosto

21 settembre -28 settembre - 26 ottobre -16 novembre - 23 novembre

$1891-9$ gennaio

The Electrician and Electrical Engineer - Londra

1887 - 18 novembre

1888 - 6 gennaio -23 marzo - 6 aprile - 20 aprile $\quad-4$ maggio

18 maggio - 25 maggio - 8 giugno - 15 giugno - 10 agosto

17 agosto -26 ottobre

The Telegraph and Telephone Journal - Ottobre 1924 - Londra 


\section{Autori e pubblicazioni diverse}

A. G. BELI, - The telephone - A lecture entiled researches in electric telephony London 1878 .

LUIGI BARZINI - Una rivendicazione - Chi inventò il telefono - Il Corriere d'America (7-I4 2I-28 febbraio 1926 - 7-I4 marzo 1926) - New York.

UMBERTO BIANCHI - La rivendicazione di una Gloria Italiana - (Antonio Meucci) Roma 1923.

DotT. Italo Brunkli. - Il Telefono - Conferenze alla R. Scuola d'Applicazione degli Ingegneri di Roma - Tip. Elzeviriana Roma 1900.

I. BRUNelli ed E. Longo - Trattato di telefonia - (Scotti) - Roma 1906.

FRANCESCo SARVognan di BRAzzA - Tre grandi inventori italiani misconosciuti II. A. Meucci - (La nuova Antologia) Roma - Ottobre r927.

A. DU MONCEL - Le Téléphone, le Microphone et le Phonographe - Paris 1880.

RINALDo FERrinI - Fisica Tecnologica - Elettricità e Magnetismo - (Hoepli) Milano 1878 .

Dr. Ing. E. FEYERABEND - so Jahre Fernsprecher in Deutschland - 1877-1927 Berlin 1927 .

I. E. KingsBury - The Telephone and Telegraph exchanges - London 1915.

D. Prccoli - $I l$ telefono - Ulrico Hoepli - Milano r884.

GEORGE B. PRESCOTT - The speaking telephone electric light and other recent electrical inventions - New York 1879

T. TIBALDi - Innocenso Manzetti di Aosta - Appunti e spigolature - (Roux Frassati e C.) Torino 1897 .

Per ricerche di biblioteca e traduzioni sono dovute grazie ai Sigg. Comm. Emilio Diena Bibliotecario - Cav, Salvatore Clemente Capo Sezione M.ro P. P. e T, T, e Sig. Lovenzo Respighi. 
APPENDICE II

\section{ALCUNI AUTOGRAFI DI A. MEUCCI}

Furono esposte alla Esposizione Nazionale della Storia della Scienza, in Firenze alcune lettere autografe del Meucci, presentate da una di lui pronipote Sig.na Bianca Meucci, e poi offerte in omaggio all'Istituto Superiore Postale Telegrafico e Telefonico per interessamento del Prof. Banti e del Direttore dei Telegrafi a Firenze Comm. Baldacci.

Queste lettere datate dall' America dal 1859 al 1886 e dirette al fratello Giuseppe a Firenze trattano di cose famigliari, e di affari, ma non contengono purtroppo nulla che si riferisca alla invenzione del telefonn, all'infuori di qualche vago accenno di speranze, di delusioni e di rammarico delle sue miserevoli condizioni.

Citiamo alcune frasi caratteristiche:

Clifton 20 dicembre 1859 . - . Sono obbligato a lavorare in una fabbrica di candele in mia vechiaia come un nero ed a soli $\mathrm{r}_{5}$ scudi alla settimana, giornata che quà si dà a un facchino, e per vivere sono obbligato a far questo s.

Slepleton Staten Island N. Y. I6 aprile 1863. - * Sarete sempre poveri nelle vostre speculazioni e sempre in mano di stranieri che fanno le speculazioni per voialtri. Unitevi alle arti e al commercio, e sarete felici, niente manca all'Italia mi dispiace che sono vecchio $\gg$.

New-York 3r marzo 1864. - saluta tutti di famiglia e parenti e a miglior fortuna mi ricorderò s.

La lettera è scritta sopra una Circolare Commerciale in quattro lingue per lo smercio di una vernice patentata dal Meucci \& per l'uso di colori e per preservare il legno dalla corruzione ed impedire agli insetti di corroderlo. »

Staten Island 25 febbraio 1880 . - È l'originale della lettera già citata in questa memoria a pag. 12: « Sai e sanno tutti bene quanto ho lavorato, e quanto lavoro ancora, ecc s.

2 agosto I88o. - « Sperate in bene: se gli affari mi si accomodano sarete felici anche dopo la mia morte.

6 dicembre I886. - a Gli affari miei non sono ancora finiti: speriamo che venga alla luce e fine avanti la mia morte *. 
Da una lettera indirizzata al fratello Giuseppe, datata da New York il 27 Agosto 1864 e pervenutagli a Firenze il 29 Settembre successivo si è ricavata la firma del Meucci quì riportata a pag. 5 in calce al ritratto.

È poi interessante una lettera presentata in due minute che si completano e rettificano l'una con l'altra, con le quali il fratello Giuseppe scrive da Firenze nel 1891 al Direttore della \& Rivista Italo-Americana , di N. Y., per avere notizie sulla eredità del fratello Antonio.

Chiede tra l'altro, Giuseppe Meucci, di s sapere con precisione se era definitivamente decisa da quei Tribunali, la questione che aveva Antonio suo fratello contro la Ditta Bell per la rivendicazione di privativa d'invenzione del telefono perchè non voleva pregiudicarsi lasciando trascorrere del tempo, ma non aveva avuto nessuna notizia neppure degli esecutori testamentari Lemmi e Bertolino ».

Come abbiamo accennato, nel groviglio delle cause sulle patenti del telefono, una sentenza della Corte degli Stati Uniti in data 21 luglio 1887 riconosceva la validità della patente Bell. Viceversa altra sentenza del novembre i 888 della Corte Suprema decideva per il proseguimento della causa intentata dal Governo degli S. U. per l'annullamento della patente con la motivazione di presunta frode ed irregolarità, e che Bell non era il primo inventore del telefono, e demandava il giudizio ad altro tribunale.

Alla data dunque del 1891 che non è precisata nella minuta, non vi era ancora aleuna decisione definitiva sulla rivendicazione reclamata dal Meucci, e probabilmente è venuta a mancare la sanzione legale essendo la questione per fatalità di cose caduta in abbandono. 